\title{
The DynaMICCS perspective
}

\section{A mission for a complete and continuous view of the Sun dedicated to magnetism, space weather and space climate}

\author{
S. Turck-Chièze • P. Lamy • C. Carr • P. H. Carton • A. Chevalier • \\ I. Dandouras - J. M. Defise - S. Dewitte · T. Dudok de Wit • \\ J. P. Halain · S. Hasan · J. F. Hochedez • T. Horbury • \\ P. Levacher • M. Meissonier • N. Murphy $\cdot$ P. Rochus • \\ A. Ruzmaikin • W. Schmutz • G. Thuillier • S. Vivès
}

Received: 13 March 2008 / Accepted: 19 June 2008 / Published online: 29 August 2008

(C) The Author(s) 2008. This article is published with open access at Springerlink.com

\begin{abstract}
The DynaMICCS mission is designed to probe and understand the dynamics of crucial regions of the Sun that determine solar variability, including the previously unexplored inner core, the radiative/convective zone interface layers, the photosphere/chromosphere layers and the low corona. The mission delivers data and knowledge that no other known mission provides
\end{abstract}

Acronym: dynamics and magnetism from the inner core to the corona of the Sun.

With the participation of Alenia Space and of the scientific team listed Section 11.

S. Turck-Chièze $(\varangle) \cdot$ P. H. Carton

CEA/DSM/ IRFU CE Saclay, 91191 Gif sur Yvette Cedex, France

e-mail: cturck@cea.fr

P. Lamy $\cdot$ P. Levacher $\cdot$ S. Vivès

Laboratoire d'Astrophysique de Marseille BP 8, 13376 Marseille cedex 12, France

C. Carr · T. Horbury

The Blackett Laboratory, Imperial College London, London, SW7 2BW, UK

A. Chevalier · S. Dewitte · J. F. Hochedez

Royal Meteorological Institute of Belgium, Ringlaan 3, 1180 Brussel, Belgium

I. Dandouras

CESR, CNRS, Toulouse, France

J. M. Defise · J. P. Halain · P. Rochus

Centre Spatial de Liège, Avenue du Pré Aily, 4031 Angleur, Belgium

T. Dudok de Wit

LPCE/CNRS, 3A Av. de la Recherche Scientifique, 45071 Orleans cedex 2, France

S. Hasan

Indian Institute of Astrophysics, Bangalore 560034, India 
for understanding space weather and space climate and for advancing stellar physics (internal dynamics) and fundamental physics (neutrino properties, atomic physics, gravitational moments...). The science objectives are achieved using Doppler and magnetic measurements of the solar surface, helioseismic and coronographic measurements, solar irradiance at different wavelengths and in-situ measurements of plasma/energetic particles/magnetic fields. The DynaMICCS payload uses an original concept studied by Thalès Alenia Space in the framework of the CNES call for formation flying missions: an external occultation of the solar light is obtained by putting an occulter spacecraft 150 $\mathrm{m}$ (or more) in front of a second spacecraft. The occulter spacecraft, a LEO platform of the mini sat class, e.g. PROTEUS, type carries the helioseismic and irradiance instruments and the formation flying technologies. The latter spacecraft of the same type carries a visible and infrared coronagraph for a unique observation of the solar corona and instrumentation for the study of the solar wind and imagers. This mission must guarantee long (one 11-year solar cycle) and continuous observations (duty cycle $>94 \%$ ) of signals that can be very weak (the gravity mode detection supposes the measurement of velocity smaller than $1 \mathrm{~mm} / \mathrm{s}$ ). This assumes no interruption in observation and very stable thermal conditions. The preferred orbit therefore is the L1 orbit, which fits these requirements very well and is also an attractive environment for the spacecraft due to its low radiation and low perturbation (solar pressure) environment. This mission is secured by instrumental $\mathrm{R}$ and $\mathrm{D}$ activities during the present and coming years. Some prototypes of different instruments are already built (GOLFNG, SDM) and the performances will be checked before launch on the ground or in space through planned missions of CNES and PROBA ESA missions (PICARD, LYRA, maybe ASPIICS).

Keywords Global solar magnetism • Formation flying - Solar activity • Solar variability $\cdot$ Solar wind-irradiance $\cdot$ Solar gravity $\cdot$ Acoustic modes

\section{Introduction}

The space missions of the last two decades (Ulysses, Yohkoh, SoHO with CLUSTER, TRACE, DOUBLE STAR) have revealed the complexity of the Sun and the impact of its variability on the earth environment (space weather).

\footnotetext{
N. Murphy · A. Ruzmaikin Jet Propulsion Laboratory, California Institute of Technology, 4800 Oak Grove Drive, Pasadena, CA 91109, USA
}

M. Meissonier · G. Thuillier

Service d'Aronomie du CNRS, 91371 Verrires-le-Buisson, France

W. Schmutz

PMOD/WRC, Dorfstrasse 33, 7260 Davos Dorf, Switzerland 
The coming SDO and Solar Orbiter missions will of course improve the present situation.

But predictions of space weather that degrade technologies, put astronauts in danger, and Climate hazards that probably affect the Earth's climate on long time scales, require uninterrupted data and a better understanding of the mechanisms of solar variability and its manifestation in the forms of flares, CMEs and the solar wind. The DynaMICCS mission provides such data and knowledge, by launching a new generation of instruments built on the experience of previous missions. It is designed to probe the solar interior and exterior. DynaMICCS explains the solar energy balance released to the Earth environment in its different forms. It enhances the capability of prediction of the Sun-Earth connecting events on daily to century time scales.

The DynaMICCS mission is aimed at delivering the knowledge of the mechanism of solar variability by detailed observation of the regions that crucially determine solar variability, from the core up to the low corona, including the characteristics of the solar wind. The DynaMICCS mission (Fig. 1) brings observations which help to get:

- an understanding of the role of the gravity waves and of the internal magnetic field in the solar radiative zone, which contains $98 \%$ of the solar mass. These quantities are believed to play key roles in the dynamics of the tachocline, the transition region between radiation and convection, which is critical for operation of solar dynamo,

- the identification of the potential different sources of dynamo in the radiative and convective regions and at their interface through the internal rotation profile,

- the building of models of the past observed great maxima and minima of solar activity (Maunder, Dalton) and some predictions for the coming decades,

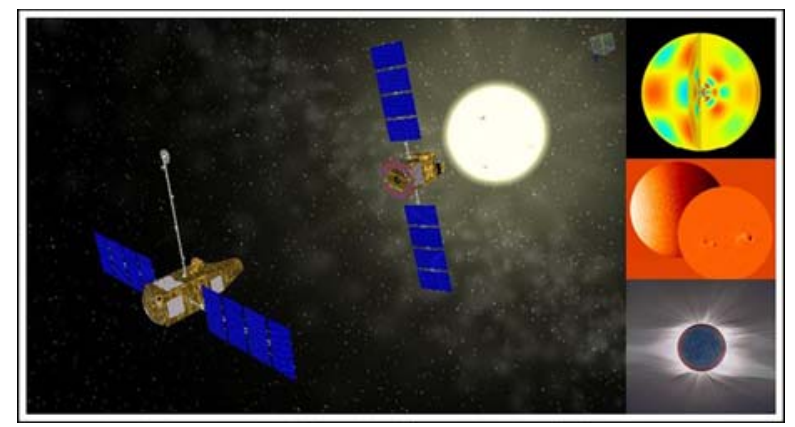

Fig. 1 Logo of DynaMICCS mission showing the formation flying spacecraft and the main objectives: (1) detection of gravity modes, (2) measurements of magnetic, Doppler and irradiance fields at photosphere-chromosphere interface, (3) imaging the complete corona. These objectives are complemented with the characteristic of the solar wind and heliospheric imagers 
- the long term evolution of the spectral irradiance to quantify the impact of solar variability on the Earth at different atmospheric heights,

- a good validation of the dynamical stellar evolution codes for solar-like stars.

DynaMICCS remotely detects internal solar acoustic and gravity modes (the first opportunity to really determine central rotation and the order of magnitude of the magnetic field) together with radius and irradiance variations from far UV to visible. It largely improves our knowledge on the transition layer between photosphere and chromosphere. Using two spacecraft separated by at least $150 \mathrm{~m}$, DynaMICCS continuously tracks the emergence of the coronal mass ejections and the slow solar wind in the low corona for the first time. Its instruments observe the Sun for a decade from an orbit around Lagrangian point L1 that ensures continuity, stability and measurements of very weak signal variations.

This document describes the main aspects of the mission following the guidance of the ESA call for the Cosmic Vision Plan 2015-2025. Several scientific documents, signed by the scientific community have been already published in [1] during the ESA process. Sections 2, 3, 4 show the richness of the scientific objectives which imply different disciplines. Sections 5, 6, 7, 8,9 describe the mission profile, the payload and the key points that we are solving to secure the DynaMICCS mission.

\section{The Sun: a typical star for a complete renewal of stellar evolution}

Magnetohydrodynamics simulations of the solar interior are rapidly developing, so observational constraints are extremely important to firmly establish its structure and dynamics as well as those of solar-like stellar interiors. It is important to recall that the Sun is always the only star for which detailed internal dynamics can be observed.

\subsection{The deep solar (stellar) dynamics}

Current used stellar structure equations include a rich microphysics and introduce to some extend rotation effects but they still ignore real magnetic field and magnetic field transport effects. We need observational constraints on the dynamical processes from the core to the surface and beyond, to build a realistic MHD description of stars and of their surrounding. For this purpose the knowledge of the dynamics of the solar radiative zone is critical. Solar observations from space can provide direct and indirect information on the solar internal dynamics and magnetic fields. DynaMICCS is the first mission to investigate the magnetic field and dynamics down to the solar core.

The SoHO mission has been successful to induce the first step towards a complete MHD vision of the Sun and to demonstrate that the solar magnetism has an internal origin. Our understanding of the solar interior evolved from 
a static picture towards a dynamical view. The meridional circulation (latitudinal flows) has been observed in the upper convective zone. The observed torsional oscillations corresponding to time variations of the solar rotation are confronted to non-linear axisymmetric mean-field dynamo model [2]. The thin tachocline, a transition region of about $5 \%$ of the solar radius located at the bottom of the convection zone where the differential rotation vanishes toward a solid rotation, plays a strong role in the understanding of the solar dynamo [3]. The sound speed profile is now very precisely established thanks to GOLF/MDI instruments $[4,5]$ down to $0.06 R_{\odot}$ but the spatial resolution is not sufficiently good in the core $\left(50 \% M_{\odot}\right)$ to get a proper determination of the density profile. The rotation profile is established (Fig. 2 left) down to the limit of the core thanks to acoustic modes, and Fig. 2 (right) shows some evidence that it could largely increase in the very inner core thanks to gravity mode first signals [6-8]. This fact needs to be confirmed by improved data.

Despite these unprecedent advances useful also for other stars, magnetic field is only directly constrained just below the photosphere, but elsewhere only upper limits are presently available. The SDO mission will largely improve our knowledge of the convective zone, but only DynaMICCS will describe the radiative zone with an unprecedent accuracy by detecting the gravity modes that diagnose the radiative zone and the inner core [9]. In the present stellar models, radiation zones are treated as stable regions. But various observations (i.e. surface abundances, sound speed and rotation profiles) sustain the idea that these zones are the seat of mixing. The most likely cause of such mixing is the radial differential rotation occurring along time which drives a large scale meridional circulation. This process has been described in a self-consistent way, namely taking into account the transport of angular momentum that modifies the rotation profile [10,11]. Series of models have been built, with rotational mixing. The agreement with observations is so largely improved for massive stars.
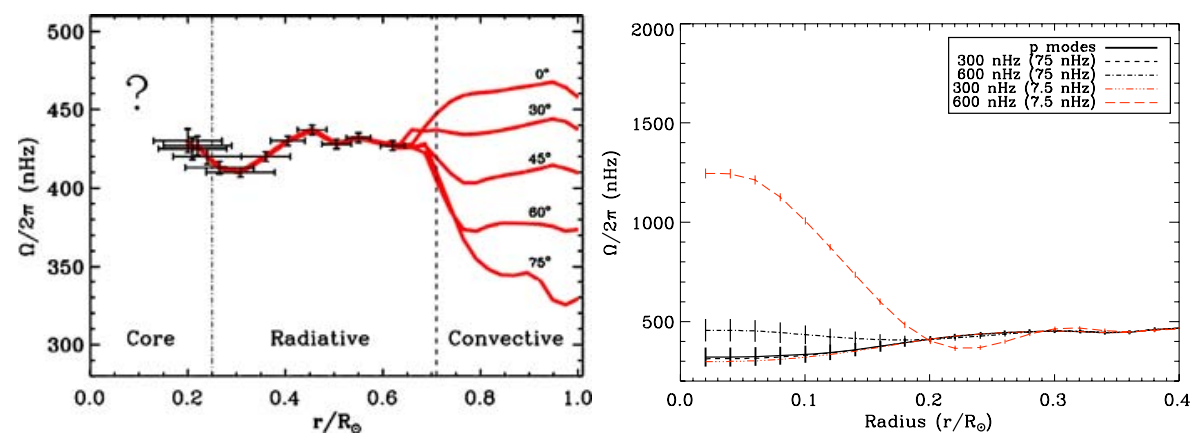

Fig. 2 Solar rotation profile deduced from the mode frequencies obtained with the GOLF and MDI instruments located aboard SoHO. From [7, 8]. The left figure uses only the acoustic modes. The right figure shows two possible solutions compatible with different interpretations of the published gravity mode candidate, the low frequency asymptotic analysis of the gravity mode range supports also an increased rotation in the solar core 
The solar flat profile for $0.2-0.65 R_{\odot}$ revealed by helioseismology (Fig. 2 left) brings a challenging information because the rotational mixing alone cannot explain this profile as shown on Fig. 3 [12]. Therefore, another more powerful process is probably operating in solar-like stars. One generally invokes the magnetic torquing $[13,14]$ or (and) the momentum redistribution by internal gravity waves which are clearly visible in all the present $3 \mathrm{D}$ simulations [15].

The non stationary problem of the radiative zone must be treated in a consistent way to take into account not only the advection of the field by the meridional circulation, but also the feedback of the magnetic field on the rotation profile and on the circulation, through the Lorentz force [16, 17]. Such interplay is illustrated by Fig. 4 left. In the case of internal gravity waves, a formalism has been recently developed describing the evolution of the rotation profile by modeling the effect of the rapidly oscillating shear layer generated by waves at the base of the convection zone [18].

Three-dimensional simulations (Fig. 4 right) have been developed to understand the complex interactions between convection, turbulence, rotation and magnetic field below the solar surface and in its upper atmosphere [3, 21]. It is the time now to develop a global model of the solar dynamics and magnetism from the deep core to the corona. It is well recognized that the tachocline plays a crucial role in the solar dynamo since it is most likely the layer where the mean toroidal magnetic field is stretched, amplified (by at least a factor of 100) and stored, until it becomes magnetically buoyant [20-22]. Spiegel and Zahn [23] showed that if there was no process to oppose its radiative spread, the solar tachocline would extend over $30 \%$ of the solar radius after $4.6 \mathrm{Gyr}$, in complete contradiction with current helioseismic inversions. They demonstrated that the anisotropic turbulence could hinder the spread of the solar tachocline to only few percentage of the radius. Elliott [24] confirmed their results numerically with a 2-D axisymmetric hydrodynamic code. However Gough and Mc Intyre [14] have proposed that the magnetic torques exerted by a weak internal fossil magnetic field could oppose the inward thermal hyperdiffusion of the

Fig. 3 Time evolution of the internal rotation profile for a solar model including only the effect of rotation in the momentum transport. From [11]

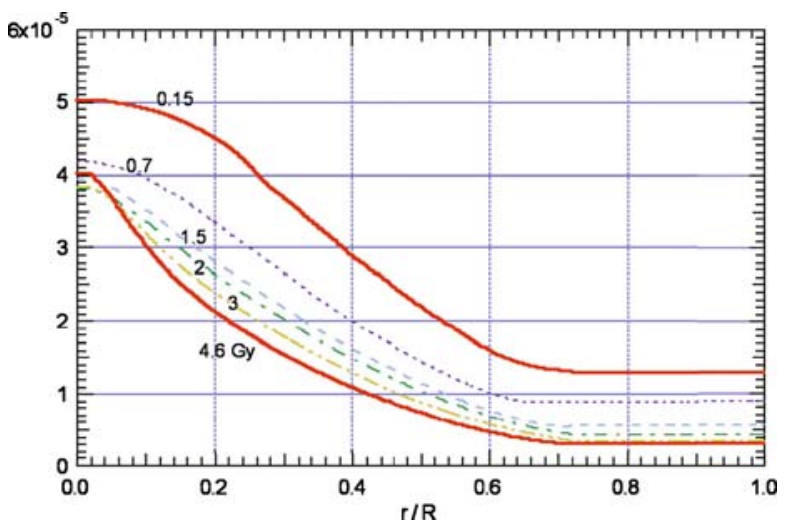



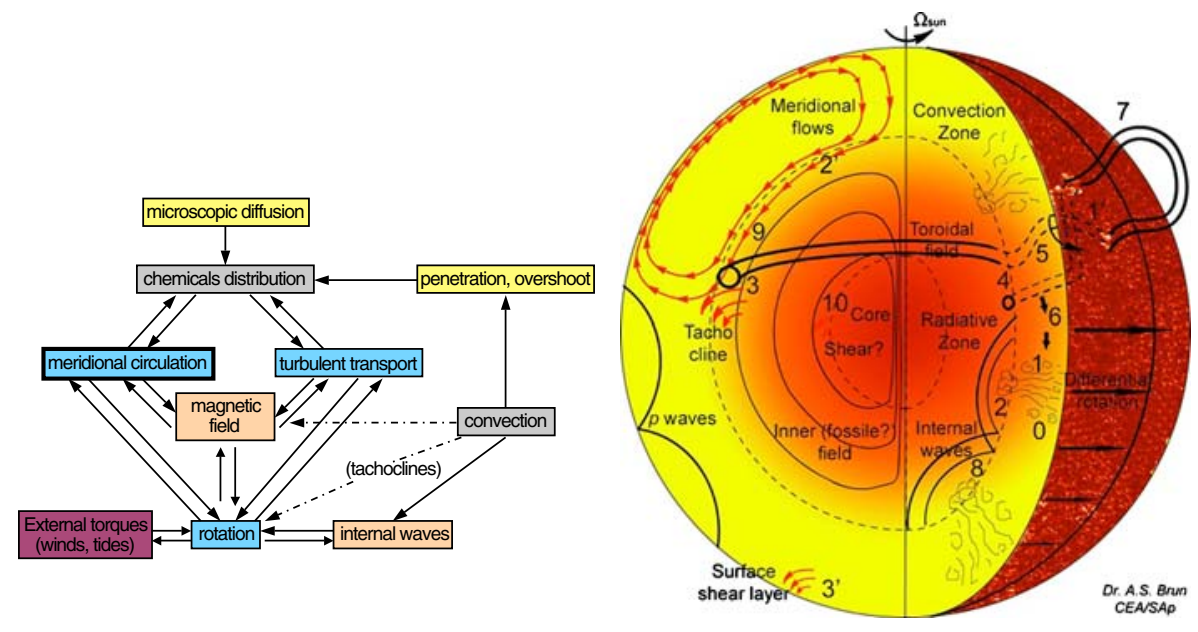

Fig. 4 Left description of the different processes that one needs to incorporate in the stellar equations in order to take into account the internal dynamics of stars. From [18]. Right a dynamical representation of the inner Sun: 0 turbulent convection (plumes); 1 generation/self-induction of $\mathrm{B}$ field ("alpha-effect") or $1^{\prime}$ tilt of active region, source of B poloidal; 2 turbulent pumping of B field in tachocline or $2^{\prime}$ transport of B field by meridional flows in $\mathrm{CZ}$ in tachocline; 3 field ordering in toroidal structures by large scale (radial and latitudinal) shear in tachocline; $3^{\prime}$ surface shear layer, solar sub surface weather (SSW), surface dynamics of sun spot?; 4 toroidal field becomes unstable to $m=1$ or 2 longitudinal instability (Parker's); 5 rise (lift) + rotation (tilt) of twisted toroidal structures; 6 recycling of weak field in CZ; 7 emergence of bipolar structures at the Sun's surface; 8 internal waves propagating in RZ and possibly extracting angular momentum; 9 interaction between dynamo induced field, inner (fossil?) field, in the tachocline (with shear, turbulence, waves, etc....); 10 instability of inner field (stable configuration?) + shearing via omega-effect at nuclear core edge? Is there a dynamo loop realized in RZ. From [19]

tachocline. A series of models showed that the tachocline could become unstable through toroidal magnetic structures embedded within it, leading to a latitudinal angular momentum transport that suppresses the shear and limits its inward diffusion [25]. With the advent of supercomputers, non linear studies become possible in the solar radiative interior and tachocline with full 3-D MHD simulations, allowing to see the interaction between the different processes [26].

Facing the numerous phenomena in presence, a real insight on the core dynamics stays crucial to investigate the role of the different mechanisms. This information must be extracted from a proper analysis of the gravity modes which are the unique probe of the central region. These modes produce low level velocity fluctuations at the surface (millimeters per second or fraction of millimeters per second) but the GOLF instrument aboard SoHO has proven their detectability [7]. Substantial improvements in their detection aboard DynaMICCS must put not only some constraints on the core rotation profile but also on the inner magnetic field due to their impact on the splitting displacements [1]. The precise determination of the central rotation and of its 
orientation axis will also put severe constraints on the initial rotation rate of the Sun and on the formation of the planetary system.

DynaMICCS will deliver the useful constraints on the solar radiative dynamics to guide these 3-D simulations.

\subsection{The transition region photosphere-chromosphere}

The acoustic modes are very sensitive to the subsurface layers but global helioseismology has not extracted any information above $0.97 R_{\odot}$. This region benefits from the development of local helioseismology. One needs to better describe these layers [46] and quantify the emergent magnetic field for understanding the evolution of the total luminosity and of the radius from one solar cycle to another one or even along one solar cycle. Recent progress came from the f-modes which represent the best tool to extract information from the subsurface region. The temporal study of these modes over the solar cycle has revealed the presence of a double sheet: the region just below $0.99 R_{\odot}$ evolves in phase with the solar cycle, although the region above seems to be in phase opposition [27, 28]. A latitudinal study of these layers is extremely useful, and an independent measurement of the photospheric radius variation along the solar cycle useful to disentangle the different actors (Fig. 5).

The CNES microsatellite PICARD, will start the measurement of global quantities such as diameter and irradiance at different wavelengths and of their variations during three years. Such efforts must be continued with DynaMICCS during a whole decade, with better observing conditions. Moreover real constraints on the thermodynamics and magnetic properties of the region between the photosphere and the chromosphere will be possible

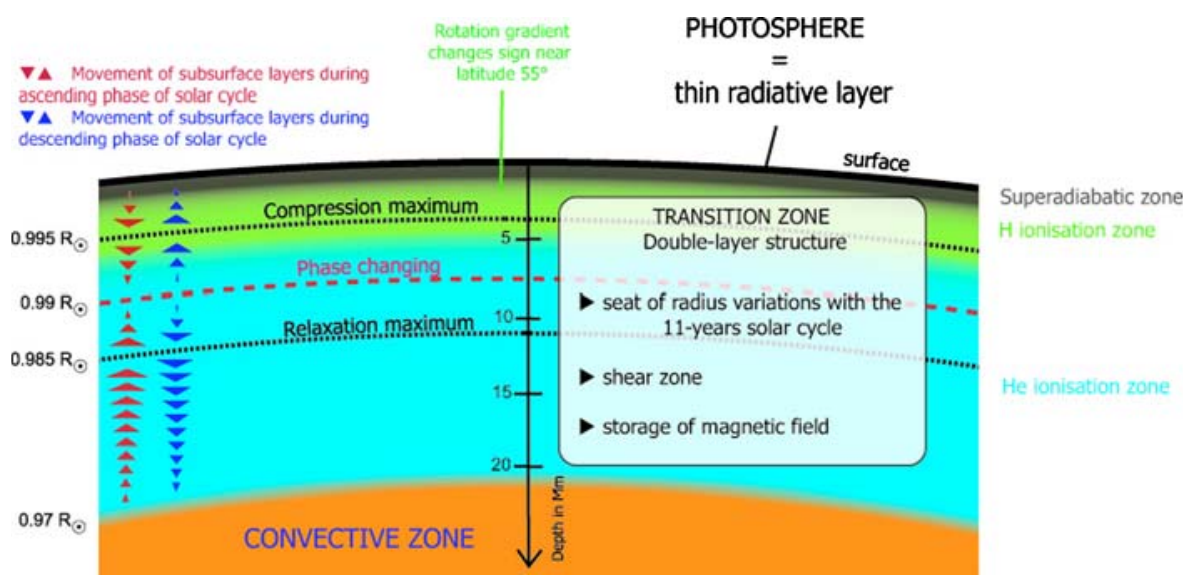

Fig. 5 Schematic view of the region below the photosphere called the leptocline (non-scale scheme). From [46]. This region is important to properly estimate how the subsurface magnetic field emerges from the photosphere and then how it evolves in the transition between photosphere to chromosphere 
through the analysis of several absorption lines: $\mathrm{He}, \mathrm{Na}, \mathrm{K}, \mathrm{Ca}$. One will see how the magnetic field modifies the density and pressure profile in the atmosphere, and we hope to identify and follow the chromospheric modes. We will also check the quality of the 3D atmospheric stellar models.

\subsection{Investigation of the dynamics and the magnetism of the solar corona}

The energy that heats the corona and accelerates the solar wind and coronal mass ejections (CMEs) originates in subphotospheric convective motions. The physical processes that transport this energy to the corona and convert it into thermal, kinetic, and magnetic energy are not fully understood. Space missions and, in particular SOHO, have greatly advanced our knowledge about coronal heating, solar wind acceleration, and CMEs, but many key questions remain unanswered. An understanding of the physical processes acting in the corona is important not only for explaining the origins of space weather, but also for establishing basic knowledge in plasma physics, directly relevant to the Sun, other stars, and astrophysical systems ranging from the interstellar medium to black hole accretion disks.

Different physical mechanisms probably govern closed magnetic loops, active regions, and the open field lines that give rise to the solar wind [29]. Both densities and volumetric heating rates decrease rapidly with distance from the photosphere, but the coronal heating rates per particle remain large in the extended corona [30,31]. This fact, combined with in situ evidence that heating continues in interplanetary space [32], implies that processes which are responsible for heating, wind acceleration, and CME ejection are dynamically important below and above the coronal base. An empirical description of the primary acceleration region (in the transition region and inner corona) is especially crucial to determine how plasma properties at $1 \mathrm{AU}$ are established.

There is a growing idea that the innermost $0.5 R_{\odot}$ of the atmosphere is dominated by different physics, which impact the extended corona. The chromosphere, transition region, and coronal base are strongly collisional and exhibit a complex magnetic topology, whereas the extended corona is relatively collisionless and uniform, with expansion of field lines into interplanetary space. Despite these local differences, the extended corona must be driven by energy that has made its way out through the lower layers. The lower solar atmosphere and corona are indeed efficiently coupled by the transport of nonthermal energy from the convection zone and by downward thermal conduction of heat from the corona. The nature of the outward energy flux is not known, but tangled magnetic fields associated with MHD waves and electric currents are believed to play a dominant role [33, 34]. The energy dissipated by tangled fields, foot point convective motions or waves (the three "theoretical paradigms") heats the coronal plasma, drives the solar wind and propulses the coronal mass ejections. Understanding this complex system requires the full characteristics of the corona, particularly in the acceleration region of the wind: one needs to measure the plasma parameters and its magnetic field. While magnetic fields emerging in the solar atmosphere control 
the structure, dynamics and heating of the solar corona, they remain essentially unattainable with the present low corona instrumentation.

Indeed, after 40 years of space coronagraphy, the lower corona $\left(<2.5 R_{\odot}\right)$ remains practically unobserved since LASCO-C1 on SOHO, aimed at observing the inner corona (with a Fabry-Perot setup), had a fairly large level of instrumental straylight, and since its Fabry-Perot did not survive the SOHO hibernation. The COR1 coronagraphs on Secchi/STEREO are simple imaging systems, with no diagnostic capability, and suffer from large instrumental straylight. Ground-based coronagraphs are affected by seeing and atmospheric conditions, and the Rayleigh scattering of the Earth atmosphere dominates the flux received by the instrument. Full Stokes polarimetry of the Fe XIII $1,074.7 \mathrm{~nm}$ line, informing on the line of sight magnetic field magnitude, were obtained only recently $[36,37]$ but are limited to bright, low altitude regions of the corona.

Performing high spatial resolution images of the corona with twodimensional spectroscopy of several emission lines from the transition region and corona base out to $2.5 R_{\odot}$ is necessary to address the following questions:

What is the relative role of convective motions and waves in the corona heating?

How are accelerated the different components of the solar wind, slow and fast?

How emerge the CMEs and how are they accelerated?

What is the strength and topology of the coronal magnetic field?

The formation flying of this mission will largely improve the present situation in showing and interpreting the different aspects of the low corona (Fig. 6) thanks to the occulter placed at $150 \mathrm{~m}$ from the coronograph. The DynaMICCS mission will permanently observe the evolution of the low corona in order to reveal how it evolves and how CMEs emerge, with in addition some unsurpassed diagnostic capability.

While observation of the heliosphere out to about $30 R_{\odot}$ has been accomplished with conventional coronagraphs, optical remote sensing observation of $\mathrm{CME}$ in the region of the heliosphere from 30 to $215 R_{\odot}$ (the Earth orbit) has only been achieved with the two Heliospheric Imager (HI) instruments on-board STEREO mission, with a nearly hemispherical sun centered field of view. In the DynaMICCS mission we have the possibility to add one or two heliospheric imagers to see how the coronal mass ejection extends into the heliosphere.

\subsection{Measurements of the solar wind}

In situ measurements of the solar wind magnetic field, density, velocity and temperature upstream of the Earth are crucial for a solar-terrestrial programme. The prime objective is to establish a connection between interplanetary perturbations and their solar source. Equally important is the 

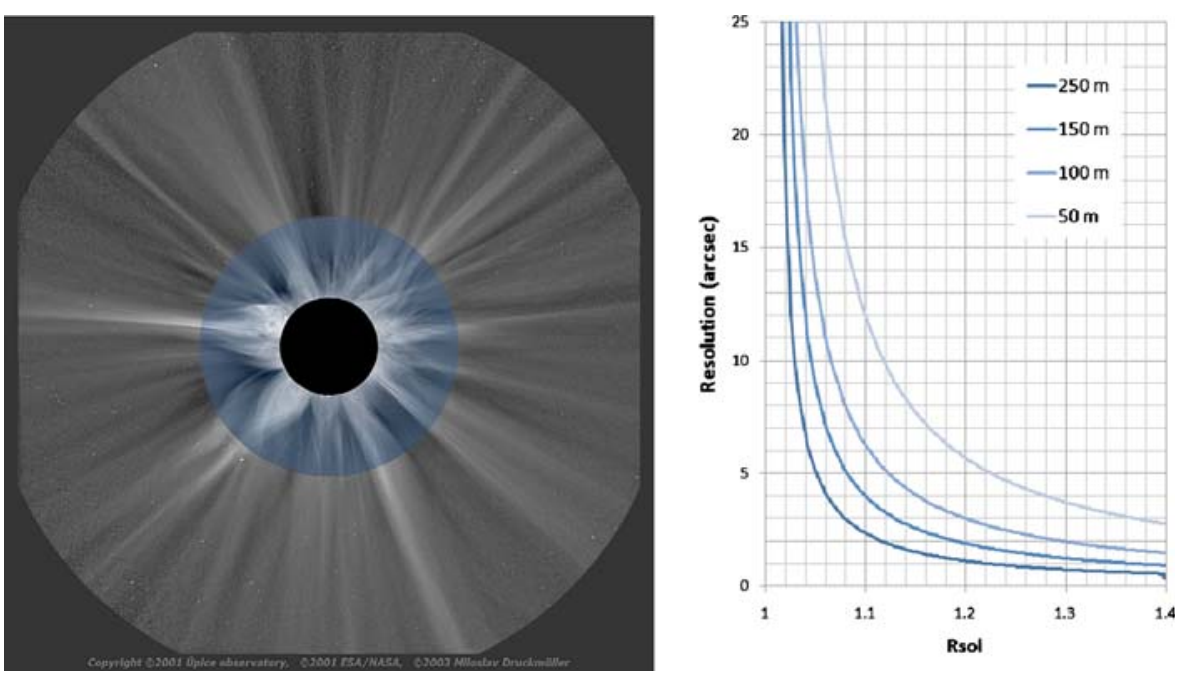

Fig. 6 Left a composite image of the corona of the 2001 eclipse in Angola. The inner part is made of the white light image obtained with a radial gradient neutral filter. The outer part is the Lasco-C2 (SoHO) image. Note the perfect correspondence of the radial fine structures. See also the same picture with other eclipses [35]. Right optical resolution of the coronograph depending on the distance between the two spacraft: one clearly sees that a distance of $150 \mathrm{~m}$ allows to reach the very low corona [68]

determination of the input parameters to the magnetosphere, namely the ram and thermal pressure of the solar wind. These quantities are today routinely measured by all magnetospheric spacecraft and also by most satellites that are located in the solar wind. Their absence on SOHO has been a real shortcoming, which was fortunately partly compensated by the presence of nearby spacecraft such as WIND and ACE. So, it is extremely useful to have onboard DynaMICCS all the complementary instruments to pursue the detection.

\section{The Sun and fundamental physics}

The knowledge of the sound speed profile in the solar core has been determinant for estimating properly the neutrino fluxes emitted by the Sun. After thirty years of experimental progress, there is a perfect agreement between the two probes of the solar interior (modes and neutrinos) [38] if oscillation parameters are used. This gives a very strong basis for determining new properties of neutrinos (masses, magnetic moments). This demonstrates that the Sun is a reference also for fundamental physics and sub leading effects may always be looked for. 


\subsection{Fundamental constants}

Over the last few years there has been an increasing interest in the possibility of varying the fundamental constants over cosmological time-scales. In fact several observations point towards the existence of a smooth dark energy component in the universe, which could be modeled via a dynamical scalar field (Quintessence). Such a cosmological scalar could couple with the terms in the matter-radiation Lagrangian, thus inducing a time variation of physical masses and couplings. Moreover, recent measurements of possible variations of the fundamental constants open the opportunity of testing the theoretical models to a good degree of precision over a wide range of cosmological epochs. Studying the time-variation of the fine-structure constant $\alpha$ is the simplest to perform. A variation of $\alpha$ affects the Coulombian interaction and therefore it would change the energy production efficiency in the stellar interior. Helioseismology can be used to monitor even tiny differences in sound speed profile in the inner region of the Sun, because the relative accuracy to which the sound speed is known is about $10^{-5}$. A small spacing frequency difference, $\delta v_{(l, n)}=v_{(l, n)}-v_{(l+2, n-1)}$ basically measures the gradient of the mean molecular weight near the center. It is an important diagnostic that puts severe constraints on the possible temporal variation of $\alpha$. The detection of the g-mode spectrum would be a key ingredient to discriminate among different theories of quintessence [39].

\subsection{Dark matter}

Cosmology, the search for the origin and nature of dark matter particles, has been intensively developed during the last decade. Numerous studies have combined in a self-consistent way the most powerful cosmic probes: the cosmic microwave background (WMAP), galaxy redshift surveys, galaxy cluster number counts, type Ia supernovae, and galaxy peculiar velocities. All these studies have led cosmologists to conclude that we live in a flat accelerating Universe, dominated by $73 \%$ dark energy and $23 \%$ cold dark matter. Although dark energy has no impact on the formation and evolution of stars, the dark matter drives the formation of the first generation of stars (population III) by influencing the collapse of overdense regions into massive stellar nurseries. Even in the absence of direct observations, cosmologists and particle physicists have been able to prove that the dark matter very likely consists of non-baryonic, long-lived, electrically neutral, weak interacting, massive particles (WIMPs). The best candidates come from the supersymmetric model of particle physics (SUSY) such as the neutralino. We now know that these particles play an active role in the formation of stars, namely, by contributing to the transport of energy inside the star thus affecting in a detectable way the present internal structure of the Sun [40]. Furthermore, if the Milky Way is populated by these particles, then they can be detected either directly in the low-background laboratory detectors or indirectly via observation of energetic neutrinos from annihilating dark matter particle pairs which have accumulated in the Sun 
and/or Earth [41]. Therefore, a better understanding of the solar interior is of fundamental importance to validate the future dark matter measurements. The research of dark matter particles within the frame of solar physics is twofold: firstly, it is interesting to select the most suitable SUSY particle candidates for dark matter that can be accommodated within a dynamical model (SDM) of the evolution of the Sun, combined with neutrino measurements at the Earth. This diagnostic will be improved in the DynaMICCS mission by the detection of gravity modes, which probe the inner core through the density profile. Secondly, the impact of these new particles on the evolution of stars will be reestimated.

\subsection{The solar gravitational moments}

The solar gravitational moments $J_{n}$ reflect the internal non-homogeneous density distribution and the non-uniform angular velocity rate. The result is a complex outer shape of the Sun, described by shape coefficients $c_{n}$, also referred to as asphericities. These quantities, $J_{n}$ and $c_{n}$, with $\mathrm{n}$ even due to axial symmetry, are related. Indeed, the solar surface is an equipotential with respect to the total solar potential (i.e. the sum of the gravitational, rotational and "magnetic" potentials). On such basis, shape asphericities, $c_{n}$, are the coefficients in a vector harmonic development of the shape of internal solar layers at radial position $r(\theta)$, with $r<R_{\odot}, \theta$ being the latitude [42, 43]. Thus, precise measurements of the solar limb fluctuations (both in latitude and with time) must contribute to get a better knowledge of the interior of the nonhomogeneous fluid body in rotation. The solar gravitational moments are at the crossroad of solar physics, astrometry and celestial mechanics. They play a key role in General Relativity. Therefore the knowledge of their precise values is fundamental. Today, the scientific community agrees on the order of magnitude of the solar quadrupole moment, $J_{2}$ of about $10^{-7}$. However, regarding the precise estimate, different methods yield different values that range between 1.6 and 6.5. For $J_{4}$, there is still a disagreement between $10^{-7}$ and $10^{-9}$. The other terms are smaller than $10^{-11}$.

DynaMICCS will largely improve the present situation by the measurements of the deep solar density profile and its shape up to the surface.

The $c_{2 i}$ curves with respect to solar depth [44-46] reveal two anomalies corresponding to two particular solar layer regions (Fig. 7) : the leptocline, at the surface, and the tachocline. While this last layer has been intensively studied these last past years, the leptocline begins just to be studied in details. It is a complex region where the turbulent pressure is important and where one observes an inversion in the radial gradient of the rotation rate at the latitude of 50 degree. Moreover it is the region where of hydrogen and helium are partially ionized [47]. Recent observations have shown that the outer shape is solar cycle dependent: the hexadecapole term $(n=4)$ seems more sensitive to the solar activity than the $n=2$ term, but this must be also confirmed through a long set of observations via space missions. 
Fig. 7 The asphericities coefficients show the distortions of the shallow layers inside the Sun, their knowledge lead to precise values of gravitational moments. Here, the structure of the tachocline and those of the leptocline, near the surface are particularly enlighten through the hexadecapole coefficient $\mathrm{c}_{4}$ coefficient. See [44, 46]

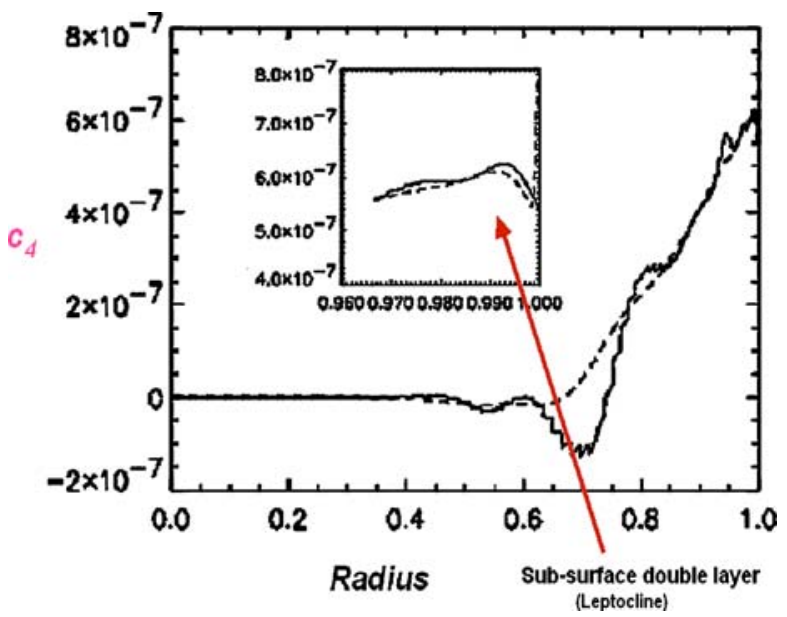

DynaMICCS will provide precise and simultaneous measurements of $\mathrm{f}$ modes, radius, central density and rotation profile and will follow their evolution over the 11 year solar cycle. This will contribute to properly determine what is the influence of the solar core dynamics and the latitudinal rotation on the values of $J_{n}, c_{n}$ and on the global solar spin $J_{\odot}$ ? A precise knowledge of $J_{n}, c_{n}$ and $J_{\odot}$ delivers fundamental information. Those parameters are not only relevant to solar astrophysics, but they have dynamical consequences in relativistic astrometry, celestial mechanics and may serve as tests for alternative theories of gravitation. In particular, the solar gravitational moments have direct influences on planetary spins and on the ecliptic plane. Through Solar System spin-orbit couplings, $J_{2}$ and $J_{\odot}$ indirectly influence the orbital parameters of the Solar System bodies. For example, the spin-orbit coupling of the Moon-Earth system propagates the influence of the solar quadrupole moment to the Moon librations and the post Newtonian parameters $\beta$ and $\gamma$ are constrained by the $J_{n}$. Space missions will decorrelate these three parameters [48]. Today $\gamma-1$ is between -2.1 and $2.310^{-5}$ and $\beta$ between 0.9998 and 1.0003 which still gives room for alternative theories at the GR.

\section{The Sun and the Earth : the radiative and magnetic connections}

The climate on earth is first driven by the radiative heat exchanges with outer space at the top of the earth's atmosphere. The techtonic plates and the earth orbital parameters lead to the great variations of the climate in the past and to the corresponding frequencies of 100, 43, 24 and 19 kyears, predicted by M. Milankovitch, and confirmed by the marine sediment data. DynaMICCS contributes to the development of Space Climate in putting onboard the measurements of the solar outputs simultaneously to the information on the internal behaviour. It also follows in time the coronal mass ejections and 
the emission of energetic particles towards the Earth to pursue the space weather information for the protection of humans and technology on earth or on its environment.

\subsection{The total solar irradiance}

The total solar irradiance (TSI) has been measured from space since 1978. Different instruments measure similar variations of the solar irradiance at different absolute levels. The instruments ERBS, ACRIM2, SOVA1, SOVA2, DIARAD/VIRGO, PMO6/VIRGO and ACRIM3 agree within $\pm 1 \mathrm{~W} / \mathrm{m}^{2}$. The early instruments ERB and ACRIM1 measured higher values but the recent TIM instrument with high target accuracy is about $5 \mathrm{~W} / \mathrm{m}^{2}$ lower than the other instruments. The accuracy of the TIM instrument is being investigated by comparison of an on-ground copy with a cryogenic radiometer at NIST. As the relative variation of the solar irradiance is the most relevant for climate change, all instruments are brought to the same absolute level and a composite TSI time series is made (Fig. 8).

In order to be able to construct this composite TSI time series, no interruption of the TSI measurements should occur. Downward spikes in the solar irradiance occur due to dark sunspots, the largest spike of the order of $-4 \mathrm{~W} / \mathrm{m}^{2}$ occurred during the solar storm of October 2003. Upward variations in phase with the 11 year activity cycle of the order of $1 \mathrm{~W} / \mathrm{m}^{2}$ occur due to bright features like facula, plages and the active network. The 11 year solar irradiance variations of $1 \mathrm{~W} / \mathrm{m}^{2}$ cause temperature variations on earth of the order of 0.1 degree (corresponding to about $0.15 \mathrm{~W} / \mathrm{m}^{2}$ on ground) but the effect of the largest fluctuations (up $4 \mathrm{~W} / \mathrm{m}^{2}$ ) is not included in this estimate. To get the long term solar irradiance variations, the difference between two 11 year activity minima is investigated. For the solar cycle 22 instrumental uncertainties existed, leading to three different estimates of the solar minimum irradiance variation. For the solar cycle 23, all the instruments except ACRIM3

Fig. 8 Composite TSI time series after bringing all instruments to the same space absolute radiometric reference (SARR) scale. From [49]

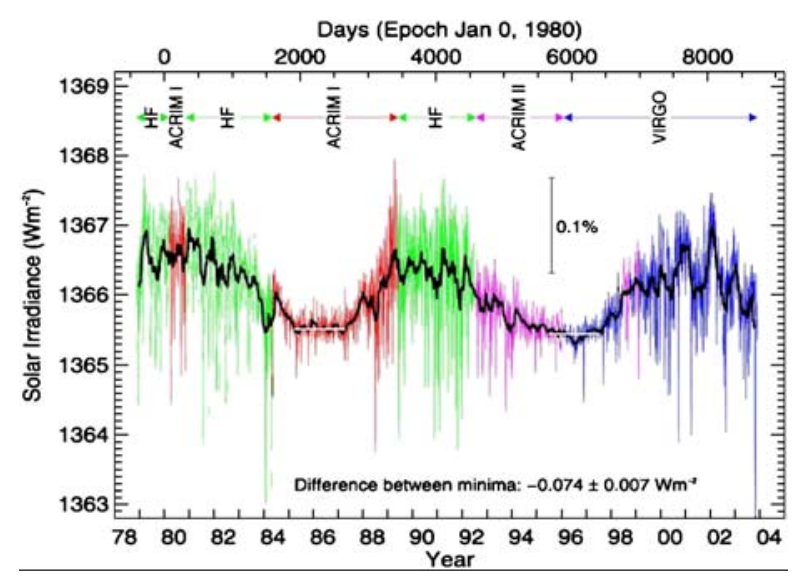


agree on the shape of the solar irradiance variation within $\pm 0.2 \mathrm{~W} / \mathrm{m}^{2}$ but SORCE produces an absolute value of the irradiance, $5 \mathrm{~W}$ lower than the other instruments. The instruments aboard DynaMICCS must reduce the uncertainty on the long-term solar irradiance variation by use of independent and simultaneous radiometers over long time periods.

There exists a long term temperature reconstruction by Moberg based on lake, ocean sediments and tree rings [50], together with a solar irradiance reconstruction by Bard et al. based on cosmogenic Be-10 concentrations in ice cores. The low temperatures during the little ice age around year 1600 correspond to a supposed low irradiance during low solar activity. The high temperatures during the medieval warm period around 1,100 correspond to a supposed high irradiance during high solar activity. This suggests the existence of a long term variation of the solar irradiance (up to $10 \mathrm{~W} / \mathrm{m}^{2}$ ) which constitutes a dominant source of climate change between 800 and 1,900.

Such a variation since 1978 is not visible. This could be due to the limited inter cycle variability for the last solar cycles or instability of the measurements. New generations of instruments on DynaMICCS will improve the quality of the measurements. The radiometers on PICARD will provide continuity during cycle 24 for the DIARAD and PMO6 radiometers on SOHO. Other radiometers that will provide continuity are SOVIM on the International Space Station and a TIM follow-on on Glory. They will maintain the observation needed for the long term solar irradiance record. DynaMICCS must insure the continuity of such measurements together with the determination of the diameter variations.

It seems that the next cycle could be predicted at least two cycles ahead. A joint measurement of the internal structure of the sun by helioseismology and of irradiance will lead to a better understanding of the variability due to the 11 year solar dynamo, and of the long-term solar irradiance variations. Presently the prediction from a dynamo simulation leads to a high amplitude cycle 24 which must be confirmed [51]. The 11 year TSI variations measured so far can be understood as modulations by dark sunspots and bright features of a constant background quiet sun irradiance. The dark sunspot irradiance has an internal origin, the bright features irradiance mainly has a chromospheric origin.

\subsection{The irradiance in the VUV}

The climatic models take today only into account the mean total irradiance variation during the 11 year cycle in excluding its strong variability during the solar maximum, it is probably a too simple scheme. Greater variations ( $10 \%$ in UV and $100 \%$ in EUV) are also connected with the Sun activity, so their impact on the ozone production in the stratosphere and on the solar modulation of the cosmic rays on the clouds in the earth atmosphere must also be studied to understand if there could be a variation (of solar or external origin) of the $77 \mathrm{~W}$ flux reflected by the clouds. The solar irradiance in the vacuum ultraviolet (VUV, 10-200 nm) range is an important quantity for solar- 
terrestrial physics and for space weather, as it generates the ionosphere and impacts the upper atmospheric layers. Although the total energy input in the VUV range is orders of magnitude below that of the total solar irradiance, its variability is much stronger, ranging from a few percent at $200 \mathrm{~nm}$ to more than $100 \%$ at smaller wavelengths. This flux is highly variable, both on long (solar cycle) and short (solar flares) timescales. A continuous monitoring of this flux is of prime importance for specifying the ionosphere, which in turn is needed for several space weather applications, such as the modelling of satellite drag (orbitography) and of radio wave propagation (telecommunication and positioning). Finally, the VUV flux is also expected to impact climate [52]. For all these reasons, there is today a high demand for continuous, spectrally resolved, and radiometrically calibrated measurements of the VUV flux.

A detailed analysis of the connection between the total solar irradiance and the VUV irradiance is especially importance for understanding what causes the variability of the former. The variability in the VUV range is expected to contribute to about $30-40 \%$ of the total solar irradiance variation, but the underlying connections are still only understood. Although solar magnetism plays a major role, the relative contributions of plages, faculae, sunspots still remain to be elucidated. DynaMICCS offers a unique opportunity to compare both the very short term $(<1 \mathrm{~s})$ and long term ( $>1$ year) variability in total and VUV irradiance.

Continuous and spectrally resolved measurements of the VUV flux have been seriously lacking so far, leading to a so-called EUV hole that has just started to be filled up by the TIMED mission [53]. VUV measurements must be carried out outside of the atmosphere and all instruments suffer from degradation. The TIMED/SEE spectrograph has been measuring the VUV flux since 2002 and will soon be replaced by SDO/EVE. Because of the finite lifetime of these instruments, most users rely today on solar proxies (e.g. Mg II index) and/or bridge the data gap with models [54]. An alternative to this would be to use an array of photometers with different bandpasses rather than a fullfledged spectrograph. Photometers offer a much lower integration time and better resilience to degradation, at the expense of a coarser spectral resolution. As shown recently $[55,56]$, one can reconstruct the full VUV spectrum with sufficient radiometric accuracy from the measurement of 4-8 properly chosen spectral bands. The reason for that comes from the remarkable coherence between the time evolution of different spectra lines.

One of the main outputs of DYNAMICCS is a continuous monitoring of the solar VUV flux, an important input for ionosphere/thermosphere specification models, and also a key parameter for solar physics studies.

\subsection{The solar magnetic cycles}

The rotation associated with the convection induces large scale circulations and shear which maintain and amplify the magnetic field in the external layers of the Sun. This drives the mean-field (11 year) dynamo: the poloidal field is transformed into toroidal field, which is amplified and stored in the 
tachocline region and then the poloidal field is restored through the action of helical convective motions. The radial differential rotation appears quickly when the surface is slowed down by the dissociation of the star from its disk. It produces different instabilities: the secular and the dynamical shear instabilities, the baroclinic and multidiffusion instabilities, which induce the transport of angular momentum and species through turbulence, the action of the centrifugal forces, the Lorentz force and the gravity waves. In the radiative zone, the rotation interacts probably with the fossil magnetic field to produce instabilities which reduce and flatten the rotation profile. Analytical calculations and 3D simulations begin to simulate them. As mentioned before, the rotation interacts also with the gravity waves that propagate in the radiative zone.

Today we begin to understand the mean-field dynamo effect reversing the field polarity every 11 years, but nobody knows whether this phenomenon induces greater cycles like the Suess (about 211 years) or Gleissberg (about 88 years) noticed variabilities. One does not know if some core dynamo exists or and if the noticed longer cycles are real and could come from deeper phenomena. Thus no prediction at the century scale is currently possible. Nevertheless one observes a large variability in the amplitude of the different solar cycles with clear effect on aurorae and apparently some connected variability on the earth climate (Maunder minimum for example), which have not been explained.

The hypothesis regarding possible core dynamo action will be checked with the DynaMICCS mission. The gravity waves could play also a potential role in cyclic phenomena. These waves are the travelling counterpart of the gravity modes. Their characteristics and propagation are described in [15]. They are excited at the base of the convective zone but also in the convection zone by the Reynolds stress. These waves are very efficient in the transport of the angular momentum during the first stage of stellar evolution. This is due to the fact that the flux of the prograde and retrograde waves differ when the rotation of the core is faster than the rotation of the convection zone. The local frequency of prograde waves diminishes, enhancing dissipation, while the corresponding retrograde waves enhance dissipation in a different place. This phenomenon produces an increase of the differential rotation locally and creates a doublepeaked shear layer which oscillates in presence of shear turbulence. This oscillation has been studied by [57] to describe the dynamical processes in young clusters. One needs to clarify if such "shear layer oscillation" SLO (name given by analogy with the atmospheric QBO) could still be active in the present Sun. If it is the case, the process can play some role in generating the fundamental cycles discussed above. To this point, detailed density and rotation profile in the core are needed.

DynaMICCS provides the quantitative values of the different variabilities and key constraints on the origin of these variabilities. This global information will quantitatively establish the past, present and, hopefully coming, role of the Sun on the Earth climate. 


\section{The mission profile}

In the previous sections, we have argued on the complementary information that DynaMICCS provides to build and control a real 3D dynamical vision of the Sun. It supposes a significant number of instruments and a permanent eclipse of the Sun. The formation flying configuration of DynaMICCS allows, for the first time, this permanent eclipse and an important payload distributed on two spacecraft (Fig. 9 left). The DynaMICCS satellites rely on rather mature technologies, the reuse of the Thales Alenia Space PROTEUS platform for the two satellites will also benefit from L2 mission heritage (HerschelPlanck) for its specific X-band communication subsystem that would replace the existing S-band telemetry and telecommand subsystem. Once the size of the platform is determined, it fixes the distance between the two satellites, which is in this case about $150 \mathrm{~m}$.

In this configuration the coronagraph can image the low corona like in a real eclipse. The stability of the configuration allows constant and continuous observations of this part of the corona, in addition to observations performed by other instruments placed on the occulter for seismic and irradiance measurements, and on the second satellite for the solar wind and heliopheric imagers.
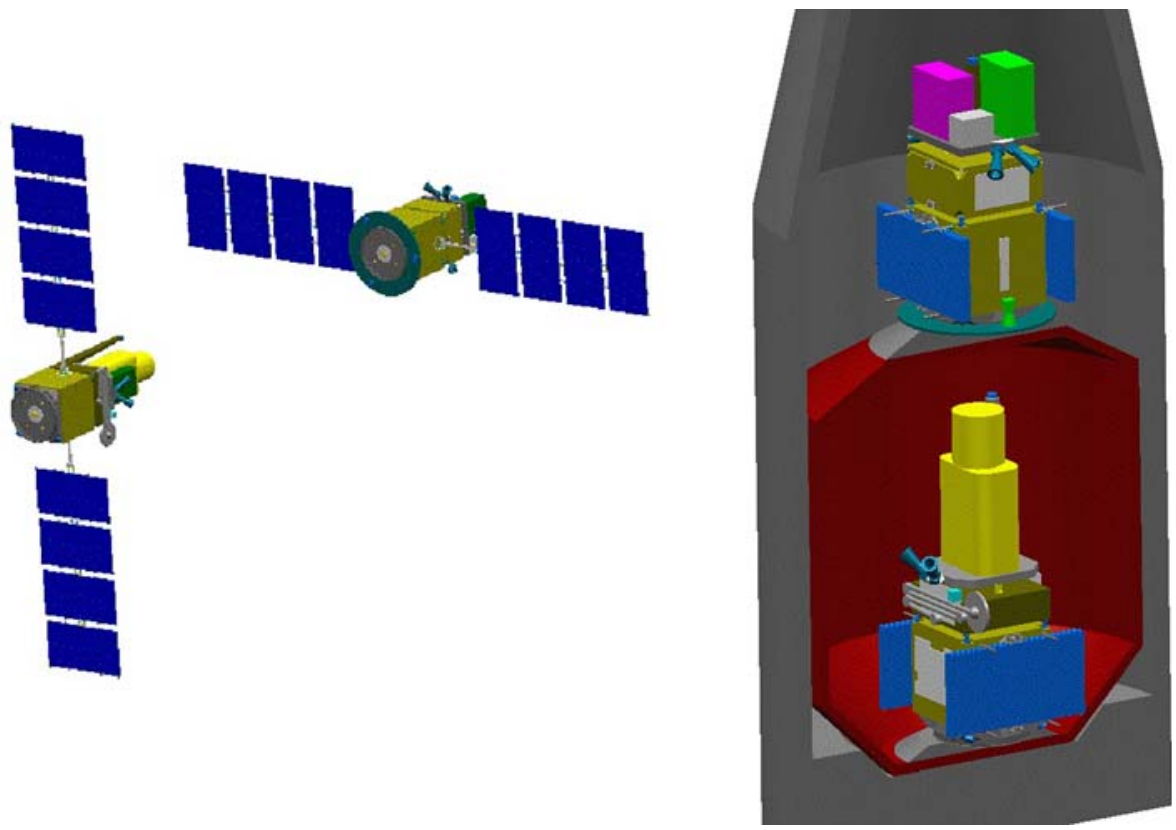

Fig. 9 Left the two satellites of the DynaMICCS mission organised in a formation flying to guarantee a permanent eclipse of the Sun for the coronograph of the second spacecraft. Right the two satellites inside the Soyuz rocket before the launch 
The proposed configuration allows accommodation of many instruments at a reasonable cost.

As a baseline, we propose to consider a halo orbit around the L1 Lagrangian point of the Sun/Earth system, like for SoHO. The main advantages of this orbit are:

- the L1 point is an ideal observatory for Sun studies, no eclipse occurs, which allows continuous observation. This point is very important for space weather studies and for helioseismology. Stable thermal environment is ensured for instruments, which eases the thermal control and the pointing performances (no thermo-elastic distortion from eclipses),

- this orbit is attractive for the spacecraft environment and control. The relatively low level of radiations allows to privilege a long life time (11 years at least targeted for DynaMICCS, like in the case of SoHO) with 5 years nominal and the other 5 or 6 years as an extension. The low level of external perturbations (main one being the solar pressure) allow to optimise the propellant budget used for attitude control and for formation flying. The absence of eclipse is also beneficial to the platform (maximum power collection, relaxed thermal and thermo-elastic constraints). The orbit injection cost in term of propellant can be low for the spacecraft, provided the halo orbit amplitude is large enough. The periodical station keeping manoeuvres induced by the halo orbit perturbations require less than $5 \mathrm{~m} / \mathrm{s}$ per year.

\subsection{The launch requirements}

The total payload mass (around $340 \mathrm{~kg}$ ) as well as the need for two spacecraft to build the formation calls for the use of the Soyuz/Fregat launcher although other launch vehicles may provide the required performance, but either at a higher cost or from non-European source. This Soyuz version allows to inject a total mass of a bit more than 2 tons, including adaptors, in a trans-L1 orbit. This allows to have some margin on the mission mass (see satellite part) and to consider the implementation of additional instruments. Moreover, the fairing dimensions ( $3.8 \mathrm{~m}$ diameter) is large enough to consider several options for the two spacecraft stacking. The stacking as a composite, or as separate spacecraft using the Soyuz support structure, is a main design driver to be traded (Fig. 9 right). Using a direct injection in the trans-L1 orbit allows to minimise the delta $\mathrm{V}$ capability of the spacecraft. Typically, an injection in a halo orbit with a semi major-axis of about 700,000 km requires less than $50 \mathrm{~m} / \mathrm{s}$ total delta $\mathrm{V}$ after a trip of about 100 days, which allows to use classical chemical propulsion systems.

\subsection{Ground segment}

The DynaMICCS mission will rely on one or several ground stations of the ESTRACK network, hosting antennas of 15 or 35 m diameter (or any 
other Deep Space Network antenna(s) from cooperative countries). The $35 \mathrm{~m}$ Cebreros antenna will offer about $8 \mathrm{~h}$ of downlink-uplink time per day to the spacecraft during nominal operations. If required, this time may be increased by using one or two more antennas at other locations, but at the expense of a significant increase of the operations cost.

\section{The proposed payload}

Twenty years after the SoHO mission approval, our capability of diagnostics has been substantially improved. After solving important questions on the solar interior, we attack today new objectives on the deep magnetism and Space Climate understanding with potential predictions, and we keep the useful space weather watchdog survey that we have for more than a decade. The main objectives of DynaMICCS are not covered by the STEREO, SDO and solar orbiter missions. The microsatellite PICARD will experience for a short time some aspect of the DynaMICCS payload (a measurement of the solar radius variation, radiometers and some seismic diagnostics). DynaMICCS is the first mission which puts together the complete set of instruments to definitively establish the 3D dynamical nature of the Sun, with enriched consequences for numerous disciplines.

6.1 The radiative and the photosphere-chromosphere regions: GOLFNG/SODISM

The dynamics of the core and of the whole radiative zone will greatly benefit from the detection of several individual gravity modes. Helioseismic instruments aboard SoHO have shown the superiority of the Doppler velocity technique for the detection of a large range of modes (Fig. 10 left). Gravity mode detections by GOLF/SoHO put the first constraints on the rotation of the core, but the solar granulation is the main source of noise in the range below $1 \mathrm{mHz}$ [59]. Since the performance of the SoHO instruments is limited for a sufficient scientific return from these modes, the signal to noise ratio must be improved by a factor 5 to 10 in the range of gravity modes. This is achieved by reducing the solar noise and by amplifying the gravity mode signal in increasing the counting signal and the sensitivity of the instrument. Recent instrument prototypes take benefit of the knowledge of the different existing techniques. Due to the tiny amplitude signals, it is extremely useful to have different instruments on board approaching the same kind of modes in different ways. It is why we propose three seismic instruments for the DynaMICCS mission: GOLFNG, SODISM and SDM.

The GOLF-NG instrument (Global Oscillation at Low Frequency New Generation, PI: S. Turck-Chièze) measures the global velocity variations. It is in development in CEA/France in collaboration with IAC/Spain. It is described in [58] and a prototype is already operational for observing the Sun from Tenerife since June 2008 with most of the waited performances 

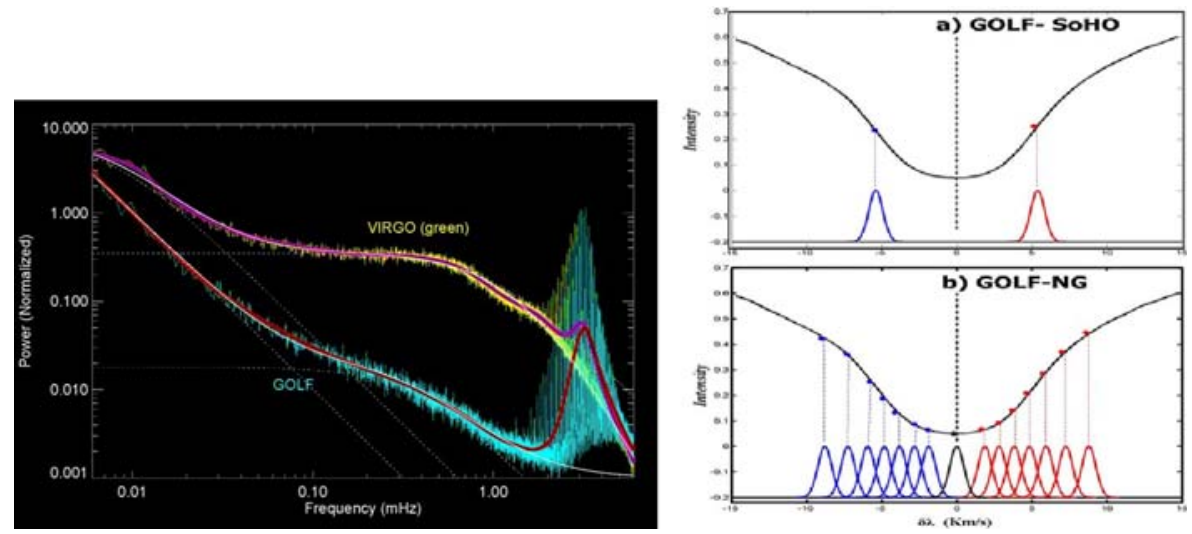

Fig. 10 Left SOHO has demonstrated the superiority of the resonant spectrometer technique in comparison with the intensity variability for exploring the low frequency range. Nevertheless in GOLF/SoHO, the solar noise is still too high to properly detect a sufficient number of gravity modes to extract the solar rotation profile in the core. Right the solar resonant spectrometer GOLF-NG principle: an alternating measurement of eight points along the sodium blue wing and then eight points along the red wing ensures a measurement of 15 points along the line in $2 \mathrm{~s}$ (or less). These different points are obtained by putting the cell inside a permanent magnet delivering a magnetic field varying quasi linearly from 2 to $8 \mathrm{kG}$. A prototype is built which already demonstrates the capability of measuring Doppler velocity at eight heights in the atmosphere of the Sun, see [59].

[59]. This instrument measures the Doppler shift of the D1 sodium Fraunhfer line by a comparison with an absolute standard given by the sodium vapour cell, the heart of the experiment [59]. A small portion of the absorption line provided by the resonance of the light in the vapour cell is split into its Zeeman components by means of a static longitudinal magnetic field the strength of which varies along the longitudinal axis in order to explore different heights of the atmosphere, from photosphere to chromosphere taking into account the fact that the patterns of the solar noise are different and consequently are non-coherent. Figure 10 right shows the principle of this new multi channel resonant spectrometer. By changing the circular polarization of the incoming flux every second, it is possible to select eight points on the right wing of the line or eight points on the left wing, with one fixed point at the center of the line. Moreover the analyzed flux has been increased by a factor 10 , in order to lower also the instrumental noise level and to allow consecutive measurements of portions of the Sun to help to the identification of the mode components. A second crystal polariser could allow the determination of the frequency power spectrum of the mean magnetic field. Figure 11 shows that if we are able to determine the different components of some individual gravity modes and to measure their asymmetric splitting, we will obtain information not only on the central rotation but also on the central magnetic field. This instrument will be also useful to check the 3D atmospheric models. 
Fig. 11 Splitting asymmetry of the components of an $\ell=1$ mode supposing a rotation and magnetic field oriented along two different axes differing from the mean rotation axis of the Sun. From [58] with the courtesy of M. Thompson

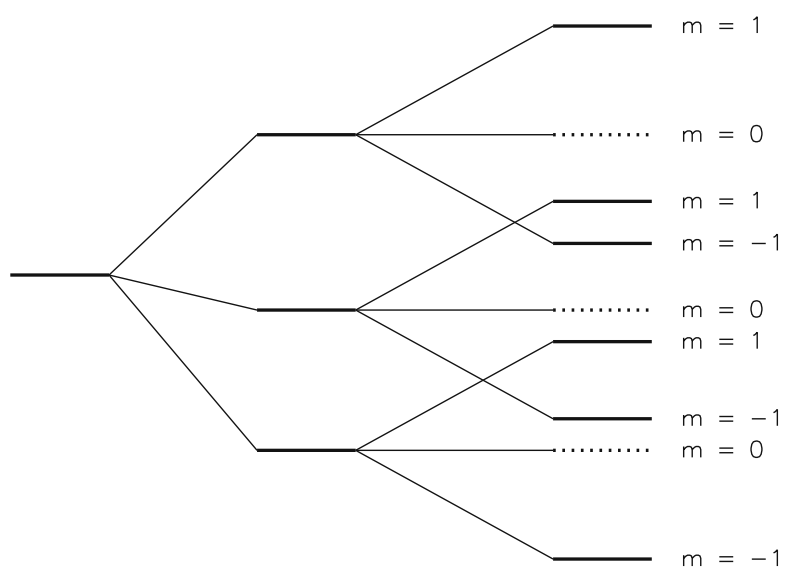

An improved version of the GOLF-NG instrument is presently under study for the low frequency range. It could join the Dome $\mathrm{C}$ site for scientific observations during PICARD mission observations. This instrument results from a 30-year expertise on resonant spectrometers used on ground (IRIS network) and in space (GOLF/SoHO). Since the gravity mode spectrum is very dense, the identification of the observed pattern components requires masks at the entrance of the instrument. Detection and identification of degrees up to $\ell=5$ for gravity modes is the objective for space. GOLF-NG will determine the core rotation profile and some estimate of the deep magnetic field. Looking continuously to the photosphere-chromosphere region, GOLFNG will check the influence of the magnetic field on these layers and determine the density and temperature profile.

The SODISM instrument might improve the mode detection by looking to the time luminosity variability using the fact that some modes are amplified at the solar limb, which will be checked in PICARD. It will carry a $170 \times 170$ macropixels of the whole Sun for intermediate degree modes. The association of GOLFNG and SODISM on the same spacecraft should improve the seismic detection of the most penetrating modes.

\subsection{The convective region, subsurface layers and radius: SODISM/SDM}

The convective zone is explored with MDI/SOHO and the same kind of observations will be continued with HMI/SDO. Local helioseismology allows to follow the fluid motions inside this region for understanding the dynamo processes. But the transition layer between the interior of the Sun and the atmosphere requires more attention since it is strongly influences solar irradiance output and its time evolution. It is important to obtain observational evidence of the extremely complex interplay between turbulence, rotation, magnetic field and microscopic phenomena in this layer. 
The analysis of f-modes improves our knowledge of the subsurface layers and shows the presence of a double sheet varying in phase (below 0.99 solar radius) or in antiphase (above) with the 11 year solar cycle $[27,28]$. SODISM/PICARD will separate the variation of the radius from the evolution of acoustic or gravity f-modes during 3 years (+extension). These measurements must be pursued in the DynaMICCS mission.

The SODISM instrument (solar diameter and surface mapper, PI: G. Thuillier) described in [60] is built for the mission PICARD. It is made of an imaging Cassegrain telescope and a CCD 2,048 × 2,048 which collects the solar images in five wavelengths: 535, 607, $782 \mathrm{~nm}$ (in the solar continuum) with an accuracy better than $10^{-3}$ arcsec for the measurement of the solar radius and asphericity, $393 \mathrm{~nm}$ for the study of the differential rotation, and $215 \mathrm{~nm}$ for the measurement of the solar activity in UV. This instrument includes an angular reference. Furthermore, from time to time, the geometrical internal scale is referred to some stellar angular distances. This instrument must follow also the $f$-modes and perform local seismology.

The simultaneous Doppler magnetograph (SDM, PI: A. Ruzmaikin) developed by JPL, USA, will produce maps of velocity and magnetic fields at several altitudes in the solar atmosphere. The strengths of the SDM lie in its wavelength stability, its narrow pass-band $(0.005 \mathrm{~nm})$, low mass and power, its compactness, and the absence of moving parts. The instrument is based on the magneto-optical filter (MOF) developed for ground observations and in use at solar observatories around the world and at the South pole [61, 62].
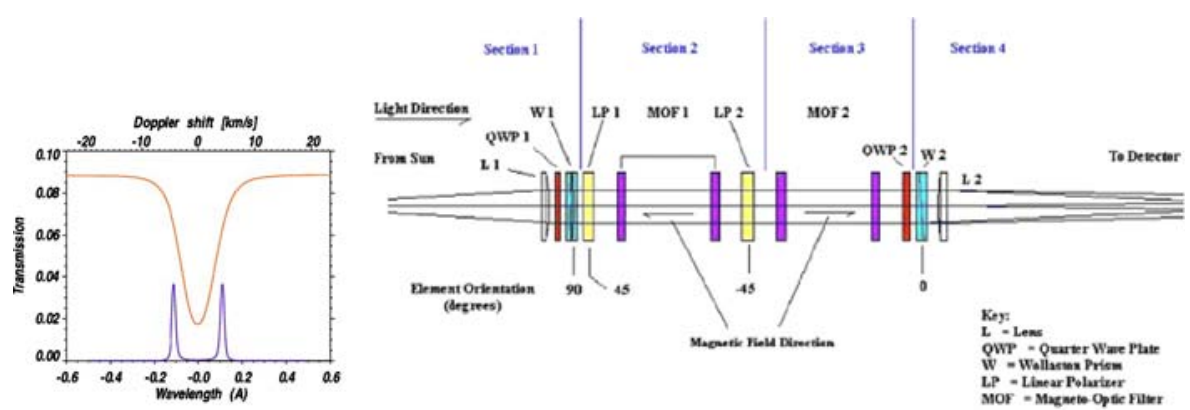

Fig. 12 a An example of the MOF passbands, compared with the K $769.9 \mathrm{~nm}$ solar absorption line. SDM will measure at least three different absorption lines in complement to the sodium line of GOLF-NG, so the two instruments will properly investigate the photosphere-chromosphere transition and will look at chromospheric modes. b Optical Design for a single MOF channel: Section 1 the polarization analyzer splits the circular polarized incoming light into two diverging beams and encodes the information about the solar magnetic field. Section 2 first MOF is put between two crossed polarizers LP, in a strong magnetic field. LP1 is at 45 to the Wollaston prism to pass both its beams with equal intensity. The linear polarized light in the two pass-bands passes through LP2. Section 3 (the wing selector). The two pass-bands are encoded with opposite directed circular polarizations by absorption in the Zeeman split absorption lines of the potassium vapor. Section 4 light leaving the wing selector is further split by the quarter wave plate and Wollaston prism into four beams, which are imaged onto the CCD detector. From four CCD frames the Doppler shift and longitudinal magnetic field are deduced 
MOF based instruments have been designed for space missions [63]. The SDM will image the Sun in the absorption lines of Na D1 at $589 \mathrm{~nm}$, K I at 770 $\mathrm{nm} \mathrm{Ca}$ I line at $422 \mathrm{~nm}$ and the $\mathrm{He}$ I $1,083 \mathrm{~nm}$, giving a range of formation heights from the mid photosphere to the upper-chromosphere. These lines sample the solar atmosphere from the photosphere to the mid-chromosphere. By acquiring simultaneous observations of the velocity and magnetic fields at several heights in the solar atmosphere SDM will be able to detect and identify the different types of magneto-acoustic waves that are present (Fig. 12). These waves will then be used to provide a detailed (seismic) mapping of both the solar atmosphere and the upper regions of the solar convection zone.

\subsection{Irradiance variability at different wavelengths: SIM/STIP/LYRA}

Time variability of solar irradiance at different wavelengths is required to better understand the climate forcing at different heights of the Earth atmosphere. The DynaMICCS mission measures spectral irradiance continuously and simultaneously using different kind of radiometers. The long-term measurements are required to determine the trend of solar irradiance, which is not properly established today. The DynaMICCS uses for this purpose a new generation of instruments that are developed based on experience acquired from $\mathrm{SoHO}$ and oncoming missions, and will produce very long and precise irradiance time series. The results will be interpreted not only using external indicators, such as faculae or sunspots, but also the information about the internal processes obtained by the DynaMICCS.

The solar irradiance monitor (SIM, PI: W. Schmutz) is provided by the Swiss Physikalisch-Meteorologisches Observatorium Davos and World Radiation Center (PMOD/WRC). The SIM will be derived from the PMO-PS type instrument which is under development for the SIM3D/solar orbiter. This new generation of solar radiometers results from a heritage of PMO6-type radiometers built for the space missions SOVA/EURECA,VIRGO/SOHO, SOVIM/ISS, and PREMOS/PICARD. The sunphotometers consist of three identical instruments, where each unit has four channels with central wavelengths at 215, 268, 535 , and $782 \mathrm{~nm}$. Today, the absolute calibration of the spectral irradiance is of the order of a few percent in the visible and up to $10 \%$ in the UV, so one needs in the future to find a way to characterize very low increase or decrease of the irradiance along decades, which justify improvements of the technique used in SoHO [64]. This is why a new generation of precision filter radiometers is presently being studied. In addition this instrument comprises two absolute radiometers which will measure total solar irradiance, continuing the series of high accuracy TSI observations of VIRGO/SOHO and TIM/SORCE. The SIM instrument on DynaMICCS will measure the total solar irradiance (TSI) with unprecedented accuracy. The SIM will be significantly lighter than any comparable TSI instrument in space. Long-term stability will be achieved with a triple-cavity design, allowing to monitor degradation, while at the same time offering redundancy. The SIM will be calibrated on ground to guarantee full traceability to the World Radiometric Reference (WRR). 
The Spectral and Total Irradiance Package instrument (STIP PI: S. Dewitte) is an improved version of the differential absolute radiometer developed by the Royal Meteorological Institute in Belgium. It is also a heritage of a continuous effort aboard SoHO, PICARD and solar orbiter to improve the accuracy of irradiance measurements. It is the successor of DIARAD aboard SoHO [65]. It is a recurrent design with gradual improvement with time, which measures the total irradiance every $3 \mathrm{~min}$ and complemented with a small bolometric oscillation sensor with a sampling period of $10 \mathrm{~s}$. STIP will use the reference channel for monitoring the ageing effect, with a target repeatability of $100 \mathrm{ppm}$ through one solar cycle. It will also allow a comparison with SIM's and other measurements like those of TIM/SORCE.

In addition to these different measurements of irradiance we add far UV measurements which appear more and more crucial due to their high variability and their impact with the earth stratosphere. Photodiodes have already been used on SOHO/SEM and on SORCE/SOLSTICE [66].

The LYRA instrument (PI: J.F. Hochedez) provided by a Belgium-Swiss consortium [67], onboard PROBA2 will be the first to test a new technology of more robust detectors with a cadence of up to $30 \mathrm{~Hz}$. LYRA is an UV radiometer which works in four UV passbands: $115-125 \mathrm{~nm}$ Lyman- $\alpha$ channel, the 200-220 nm Hertzberg continuum range, the aluminium filter channel 17$70 \mathrm{~nm}$ including He II and the zirconium filter channel between 1 and $20 \mathrm{~nm}$. This instrument will be monitored onboard by led sources. It will be extremely useful for operational space weather but also to follow the long trend in these wavelengths. All these instruments together will contribute to follow the whole solar spectrum in time during the long mission and in continuity with some others. The instrumental concept for DYNAMICCS will be a direct heritage of LYRA, with 6 photodiodes to improve the accuracy of the spectral reconstruction and new technology diamond detectors.

\subsection{Study of the low corona: VIC}

The visible and infrared coronagraph (VIC, PI: P. Lamy) is based on the ASPIICS concept [68] proposed for the PROBA-3 (ESA) technology mission and developed at LAM (Laboratoire d Astrophysique de Marseille, France) but with much enlarged scientific capabilities (Fig. 13). VIC is an externally occulted coronagraph entirely protected from direct sunlight by remaining in the shadow of the external occulter hosted by the front spacecraft. The classical design of an externally occulted coronagraph is adapted to both the detection of the very inner corona as close as 1.01 solar radius from the solar centre, and the 3D spectroscopic and polarimetric observations in two channels. The visible channel is devoted to several emission lines (Fe XIV at $530.3 \mathrm{~nm}, \mathrm{Fe}$ $\mathrm{X}$ at $637.4 \mathrm{~nm}, \mathrm{He} \mathrm{I}$ at $587.6 \mathrm{~nm}$ ) and a broad continuum band (Thomson scattering by electrons, $540-570 \mathrm{~nm}$ ). The infrared (IR) channel is devoted to the Fe XIII emission line at $1,074.7 \mathrm{~nm}$ to measure the coronal magnetic field via the Zeeman effect. An optional ultraviolet (UV) channel devoted to the Lyman alpha emission line of the hydrogen at $121.6 \mathrm{~nm}$ to measure the 
Fig. 13 Schematic view of the optical concept of the coronograph visible/IR VIC

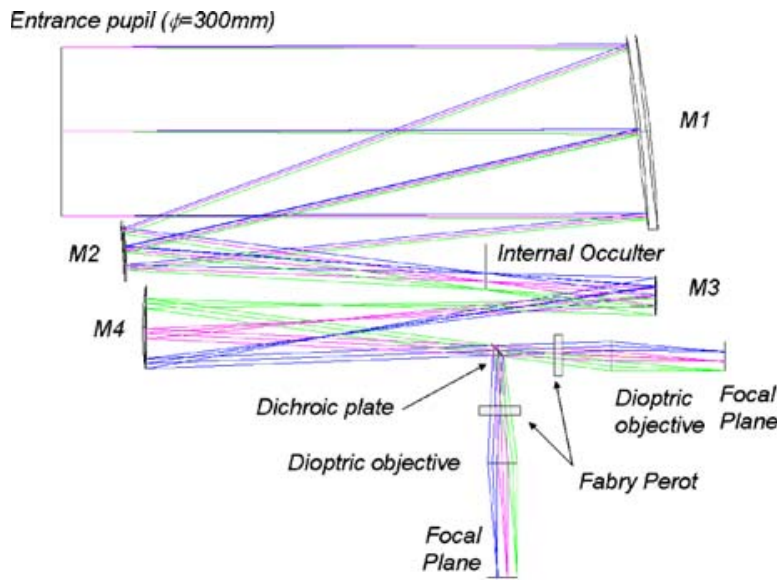

coronal magnetic field via the Hanle effect will be considered in the next study phase.

The external occulter (EO) blocks the light from the solar disk while a twoor three-mirrors telescope (300 $\mathrm{mm}$ diameter entrance aperture) collects and reimages the light coming from the corona onto the internal occulter (IO). The IO is a key element allowing to drastically reduce the stray light level by blocking the bright diffraction fringe surrounding the EO. The design of the telescope is critical because a high level of both aberrations and scattered light will prevent observing the very inner corona. A relay optics (two-mirrors configuration) forms a collimated beam and produces a real image of the entrance pupil with a magnification of about 10. A dichroic plate located in the collimated beam separates both channels. One narrow-bandpass Fabry-Perot (F-P) interferometer is located in each channel with a set of blocking filters allowing selecting a specific emission line. Polarimetric analyses and spectral selections are performed in the collimated beams. In each channel the final image is formed by a dioptric objective on a specific detector. Liquid crystal polarimeters are currently in space qualification for different space programs (solar orbiter, ESA) and are baselined for VIC assuming full maturity.

As an optional addition, an heliospheric imager (HI-D) [69] could be delivered by CSL in Belgium on board DYNAMICCS mission. It will focus on the coronal mass ejection (CME) in space between Earth and Sun. CME is a class of large-scale transient phenomena revealed most effectively in white light observations of the corona. They are characterized by explosive and outwardly expanding coronal density fluctuations that escape the solar gravitational potential well and propagate into the heliosphere. It could be interesting to follow the CME propagation through the inner heliosphere near the sun-earth line as on STEREO. Detection of the CME signal against the natural background is a strong function of elongation and a very low level of detection, $10^{-9}$ to $10^{-12}$ of the sun intensity is required. A specific baffling protection from solar disk brightness and other surrounding straylight sources 
(Earth, Moon) must be designed for this instrument. The large field of view can be shared into two separate cameras. Such imager is still under study to properly deserve the DynaMICCS objectives.

\subsection{Study of the solar wind: DM/TEM/RS/SEP}

The basic payload for the solar wind study consists of four instruments : a magnetometer, a thermal plasma monitor, a radio spectrograph and an energetic particle monitor. The interplanetary magnetic field is the most important parameter of the solar wind, as it allows to detect and identify geoeffective interplanetary perturbations and establish their connection with their emergence from the Sun. For that, the three components of the magnetic field need to be measured at the spacecraft location, using a fluxgate magnetometer, provided by Imperial College. Such instruments are routinely used on many spacecraft, such as Cluster, Ulysses, Themis, ACE, AMPTE, etc. The intensity of the field at L1 is typically of a few nanoteslas and a sampling rate of $1 \mathrm{~min}$ is sufficient. An important issue with magnetometer measurements is that of eliminating magnetic interference from other items on the spacecraft. So, the sensors are placed on a boom that is at least $3 \mathrm{~m}$ long. The data processing unit can be placed on the main spacecraft. Another important issue is the elimination of any time-varying interference, e.g. from structures whose magnetic properties change with temperature or age.

The thermal plasma monitor, provided by the CESR (PI: I. Dandouras) will provides access to the bulk plasma properties of the solar wind (density, velocity and temperature) by measuring the ion velocity distribution up to about $20 \mathrm{keV}$. A three-dimensional velocity distribution in a $45 \times 45$ degrees $^{2}$ cone centred in the main solar wind arrival direction is adequate here for ions. Since the spacecraft is not spinning, the instrument should have electrostatic scanning to sample the different directions (towards the Sun). The instrument consists of electrostatic analysers (ESAs) that measure the particle distribution functions over the energy range, from typically $10 \mathrm{eV}$ to $20 \mathrm{keV}$. There is much heritage from Cluster, Themis (for spinned spacecraft), and Stereo (unspinned). To derive bulk plasma, the basic moments of the velocity distribution can be computed on-board and converted to physical quantities on the ground. This greatly reduces the amount of data to be downlinked. Sample full 3D distributions will also be transmitted, after on-board compression, but with a lower rate than the moments. The solar wind velocity has to be derived from ion moments rather than electron moments because the typical thermal velocities of ions are 40 times smaller than those of the electrons. This instrument should ideally be located on the magnetometer boom, at $1 \mathrm{~m}$ from the spacecraft.

The monitoring of interplanetary shocks will be done by a radio spectrograph provided by the LPCE (PI: T. Dudok de Wit) from their emergence in the corona or interplanetary space up to their arrival at Earth, where they can trigger major space weather events. These shocks emit type II radio emissions that have been measured by several spacecraft (WIND, Stereo). Such electromag- 
netic waves are traditionally measured using long electric field antennae. In this frequency range ( $30 \mathrm{kHz}-30 \mathrm{MHz})$, however, magnetic field measurements offer a promising alternative, with small loop coils instead of long electric wire antennae. Loop coil are particularly interesting on a unspinned spacecraft, on which electric field measurements require heavy boom antennae. Three components of the magnetic field are needed to do direction finding. The instrument consists of three imbricated and perpendicular loop antennae $(<40 \mathrm{~cm}$ diameter). As for the magnetometer, the instrument should be placed at least $3 \mathrm{~m}$ away from the spacecraft, on the same boom as the magnetometer, while the spectral analyser can be located onboard. This instrument returns spectral power densities of the three components, together with their crossspectral densities. From this, the intensity, the polarisation and the ellipticity of the radio waves can be inferred. The heritage of this instrument is a single loop coil that flew onboard the CHARM-1 sounding rocket in 2007 (with a second scheduled flight for 2009). The deployment mechanism is presently being investigated.

Energetic ions are important tracers of acceleration processes on the Sun (solar energetic particles, or SEP) and of the interplanetary medium (acceleration by shocks, by corotating interacting regions). Their propagation is strongly determined by the large-scale geometry of the interplanetary field. The prediction and monitoring of SEPs is also a key issue in space weather. The energy range of interest is typically 1 to $100 \mathrm{MeV}$. Such ions are routinely detected with high energy particle telescopes, with a heritage on SOHO (ERNE) and Cluster. The instrument typically has five energy channels, spaced logarithmically between 1 and $100 \mathrm{MeV}$. Mass resolution is an asset for elemental and isotopic composition studies, which provide further insight into the acceleration mechanisms. Typically, four channels are needed
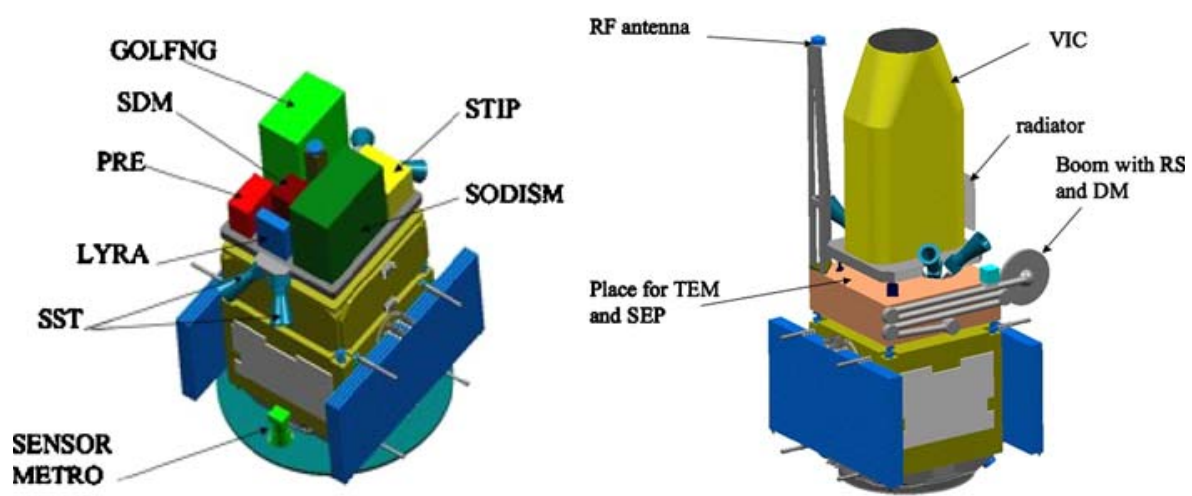

Fig. 14 The formation flying DynaMICCS mission formed by two PROTEUS platforms: the occulter spacecraft equipped with three helioseismic instruments + three irradiance other instruments, and the second one equipped with coronograph, imagers and solar wind instruments 
to distinguish protons, alphas, $\mathrm{Si}$ and $\mathrm{Fe}$. In contrast to the thermal plasma monitor, only coarse spatial resolution is needed. Since the spacecraft is not spinning, the instrument should have one or a few sensors to sample the different directions.

The characteristics of the different instruments are summarized in Tables 1 and 2 and the accommodation is shown on Fig. 14.

\section{The key factors of the spacecraft for the DynaMICCS mission}

The formation flying configuration imposes very specific design constraints to both spacecraft, with respect to classical satellites. In particular, the following specific functions must be implemented: (1) propulsion system with milliNewton class thrusters on the chaser, for formation keeping, (2) formation flying sensors suite on both spacecraft with their field of view constraints, (3) formation flying onboard software specific modules such as guidance, navigation and control, collision avoidance, (4) inter satellites link (ISL) for formation flying management (failure detection, isolation and recovery) and formation flying guidance, navigation and control, (5) virtual spacecraft concept for formation management from ground, as a single S/C during normal operations (using ISL for TM/TC transfer): this concept simplifies the operations, (6) separate communication access to each spacecraft during critical phases (deployment, safe configurations): this implies the use of multiple access techniques for avoiding RF interferences (frequency division multiplexed access, time division multiplexed access). Also, this allows to continue to manage the spacecraft independently in case of fatal formation failure, hence continuing the mission for instruments other than coronagraph.

The pointing performances are likely to be sized by the coronagraph mission: the coronagraph pointing requirement is 20 arcsec, which is achievable with recent star tracker performances. The lateral positioning induces the need to introduce a first stage of metrology. The metrology subsystem, which is already breadboarded and characterised (ULLIS) provides some hundreds of micrometers, that is an order of magnitude better than the measurement accuracy needed. The coronagraph stability requirement (about 0.5 arcsec over $15 \mathrm{~s}$ ) is also stringent and should be analysed in detail.

The L1 Lagrangian point being located at 1.5 million $\mathrm{km}$ from the Earth, the distance to the spacecraft will be even higher due to the large halo orbit amplitude. This is a main driver for the payload data transmission subsystem that will have to download a high volume of science data, making necessary the use of X-band or even Ka-band. However, considering the instruments overall data rate (a bit lower than $400 \mathrm{~kb} / \mathrm{s}$ over $24 \mathrm{~h}$ for the occulter spacecraft payload, a bit greater than $200 \mathrm{~kb} / \mathrm{s}$ over $24 \mathrm{~h}$ for the coronagraph spacecraft payload), a high gain steerable antenna, either mechanic (lower reliability) or electronic (greater cost), is required to meet the demand. In order to simplify the overall communication subsystem design, a single subsystem will 


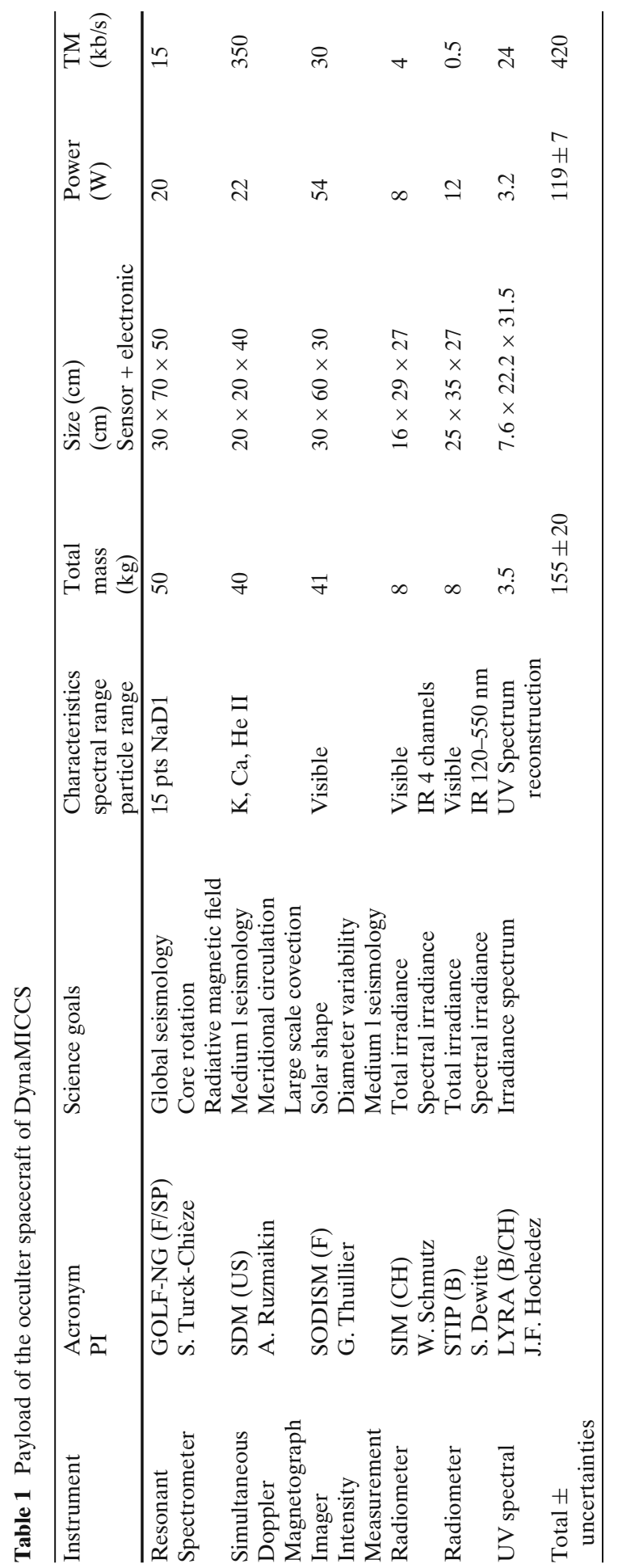




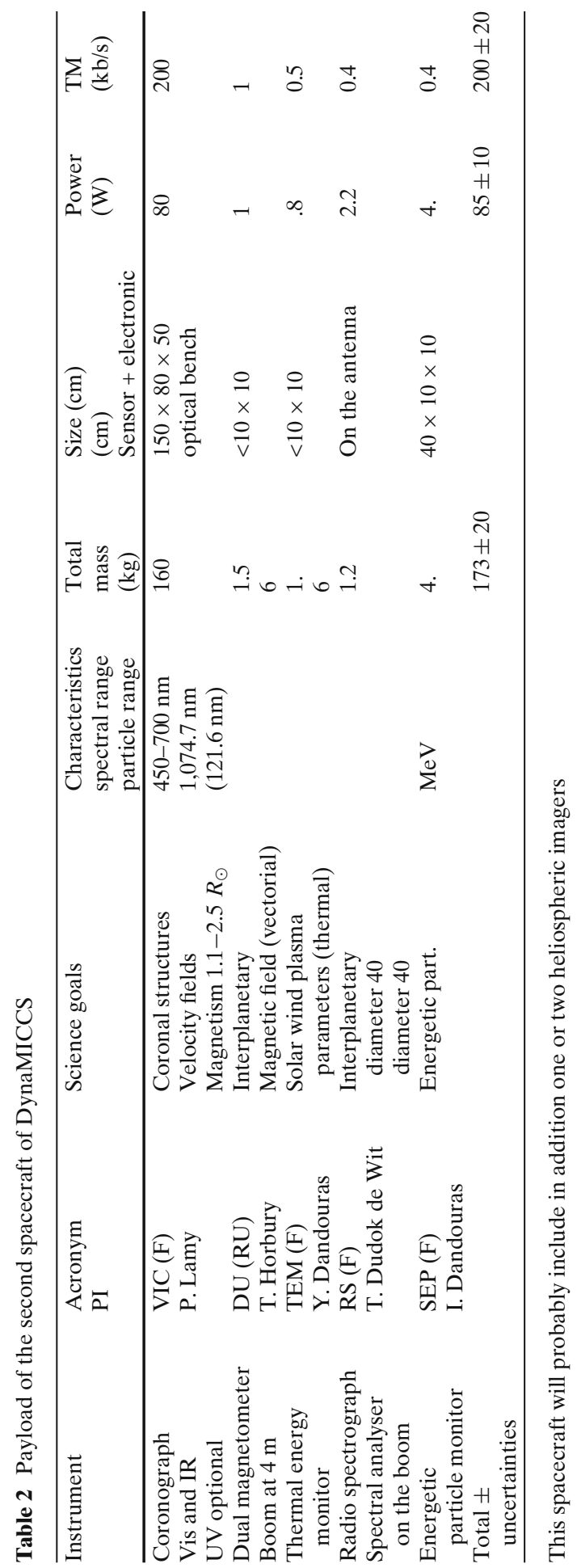


be privileged both for science data downlink and satellite data telemetry (housekeeping data) and telecommand from ground (TM/TC). The objective of space weather implies the use of several antennas: one high or medium gain antenna (HGA/MGA) for the science data download, and two or three low gain antennas (LGA) for TM/TC, ensuring redundancy and the possibility to communicate with the spacecraft whatever its attitude.

Using the Cebreros antenna and a visibility period of $8 \mathrm{~h}$ per day, the payload data rate of $600 \mathrm{~kb} / \mathrm{s}$ requires a high gain antenna with high power amplifiers $(>100 \mathrm{~W})$. Link budget with a medium gain antenna (MGA) should be refined to check with more details the compatibility with the mission. Of course, the use of a MGA would be preferable because it would avoid the high gain antenna steering capability (mechanical or electronic), and as such would greatly decrease the antenna cost as well as the risk of failure. Both spacecraft will embark this X-band (or Ka-band) communication subsystem, so that the communications redundancy is greatly improved (using the Inter Satellite Link); also, this will allow to continue the mission except for coronagraph, if the formation encounters a fatal failure.

The coronagraph spacecraft will have a single RF antenna looking front and three others located on the aft, giving it a complete $4 \pi$ steradians field of view for the launcher separation and collision avoidance manoeuvres. This $\mathrm{RF}$ system is completed with an optical metrology. The coronagraph satellite being the target, no other specific subsystem is required for the formation flying maintenance (it is however equipped with a classical chemical propulsion module for attitude and orbit control).

The occulter spacecraft will have three RF antenna looking aft toward the target for precise metrology and a fourth one to get a complete $4 \pi$ steradians field of view for launcher separation and collision avoidance manoeuvres. It will be also equipped with an optical metrology subsystem. This spacecraft acting as the chaser of the formation flying configuration, it will house a highprecision/low-thrust propulsion module to maintain the relative displacement and attitude between the two spacecraft. However, although this configuration is baselined for the time being, a different accommodation of formation flying sensors or even different types of sensors could be traded in the first phase of the study.

The DynaMICCS payload integrates 10 to 12 instruments (with possible extension). Each of them will be under the responsibility of an associated PI. DynaMICCS may be viewed as an observatory of complementary instruments very much like SoHO, and the science analysis and interpretation could very much benefit from one or several Science Data Centers. A main data center would then be directly and physically associated to the POC, while secondary data centers devoted to specific science aspects of the mission could be located in different places and countries. We propose to have one added SDC in US and another one in India (PI: S. Hasan) to demonstrate the world character of such mission. Those centers would have to be funded by their agencies and will offer adding managements for the treatment of the data. They could then host several instrument teams and take responsibility for generating Level-1 
and Level-2 data, general data distribution, data archiving and for outreach programs.

The DynaMICCS data policy is to have completely open access to quicklook data and to Level-0.5 data as soon as the routine processing steps have been completed. The data will be accessible via the Web using a searchable database. Free access to higher level data products, including the calibration data and procedures necessary to recalibrate and reprocess the data will be given after a proprietary period which will be decided by each instrument team, but which will not exceed one year.

\section{Key technology areas}

The DynaMICCS mission is relying on mature Formation Flying technologies, resulting in low mission risks. DynaMICCS would be a perfect M-class formation flying mission to prepare the large formation flying mission (XEUS, GRI, DARWIN) after the SIMBOLX demonstration. It must demonstrate European high performance formation flying capability. The launch margin could enable to embark additional metrology for in-orbit demonstration of the technology required for the next missions. Several R\&D are realized for the payload, especially the development of prototypes for GOLFNG and SDM in order to demonstrate the progress done by these instruments. Such R\&D programs are justified by the performances of the dedicated instruments which are real metrologic instruments, for example GOLFNG needs to reach a $10^{-7}$ sensitivity which is not guaranteed in a let and go mission profile. LYRA will flight on PROBA 2.

The proposed coronagraph (VIC) is the natural development and extension of the ASPIICS (Association de Satellites pour lImagerie et IInterferometrie de la Couronne Solaire) presently under study for the phase A study of the ESA PROBA 3 mission of demonstration of formation flying. VIC will implement scanning Fabry-Perot of the type which has already been flown on SOHO/LASCO. The only novel technology consists in an electro-optically modulated polarimeter which incorporates a liquid crystal variable retarder. However, this system is already extensively used for ground-based observations and its space qualification is underway for the Herschel solar experiment as well as for several solar orbiter instruments. It is therefore anticipated that the technology will be fully mature in the Cosmic Vision era.

For the spacecraft, we use the same strategy. Indeed, the main technologies necessary for the mission is the formation flying, which will be mature at the time of the phase A kick-off. Thales Alenia Space will fully demonstrate the RF metrology and the associated formation control modes and software on PRISMA. The DynaMICCS formation flying needs are fully covered by current technology development plan.

Just before the release of proposals, another solar formation flying mission, HIRISE, has been deduced from the present study using in that case the heritage of the Herschel platform. Using such a platform allows a larger 
payload mass for each spacecraft and consequently a greater scientific return, with in addition high resolution imager and spectroscopy explorer on the first satellite and an increase distance between spacecraft. The extra cost could be solved by a large collaboration between space agencies. Demonstrating that we are convinced that we can build around L1 a solar mission including global and local seismology, irradiance, coronography, spectroscopy, high resolution imagers is a very exciting perspective for the ESA Cosmic Vision 2020-2025.

\section{Communication and Outreach}

The DynaMICCS will follow the path of successful public outreach laid by SOHO and other solar missions. It will add new aspects related to space weather, space climate, stellar evolution, and fundamental physics. In this way it will affect the astrophysics and physics community in addition to the climatologists, meteorologists and geophysicists. Public will become aware of human role in space environment as it is aware now of the problems related to global warming.

Considering these different levels of information or communications, we develop, in parallel to the scientific package, a public package on the POC page web to treat the different levels of scientific return. We actually started this task by outreaching the SoHO results and related information. Some our WEB pages already exist, for example the ROB website in Belgium. We consider the development of the existing sites. The first result before the DynaMICCS mission will come from SDO and PICARD so we will immediately create these sites to inform people and also national companies who are interested by the eruptive Sun.

We also prepare some specific educational materials for schools at different level to educate children of Europe on the environmental problems and on the role of human at our planet. Such subject is also a good way to inform people about the role of science in delivering quantitative answers concerning the complex phenomena occurring between the Sun and the Earth.

DynaMICCS has high potential for outreach not only of fundamental science but also of applied science, with societal effects. The first one is space weather, for which there is increasing public awareness. DynaMICCS provides solar data (coronagraph images, real-time VUV flux) that could be used by schools in much the same way SOHO and GOES data are used today. The second one is Space Climate, which is focused on long-term changes. The prime interest here is the solar variability influence on global climate change.

\section{List of CoI}

This mission has been prepared by exchange inside a large scientific community covering more than 30 institutes during the last 3 years. The CoI list of the DynaMICCS scientific team is: J. Arnaud, LUAN, France; A. 
Ajabshirizadeh, Maragha, Iran; J. Ballot, MPI Garching, Germany; A. Benz, ETH, Switzerland; V. Bommier, LERMA, France; A. Bonanno, Italy; A. S. Brun, CEA, France; M. Casse, CEA, France; P. Charbonneau, U. Montreal, Canada; F. Clette, ROB, Belgium; S. Couvidat, HEPL, USA; T. Corbard, OCA, France; B. Dintrans, OMP, France; V. Domingo, U Valencia, Spain; A. Eff Darwich, U Laguna, Spain; P. Eggenberger, U. Genve, Switzerland; W. Finsterle, PMOD/WRC, Switzerland; R.A. Garcia, CEA, France; J. Guzik, LA, USA; G. Houdek, U Cambridge, England; S. Jefferies, Hawai, USA; S. Jiménez-Reyes, IAC, Spain; A. Kosovichev, HEPL, USA; R. Lallement, SA, France; S.Lefebvre, CEA, France; I. Lopes, Lisboa, Portugal ; D. Maia, CICGE, Portugal; S. Mathis, CEA, France; S. Mekaoui, RMIB, Belgium; P. Nghiem, CEA France; P. Palle, IAC, Spain; J-R Pacheco, SRG, Spain; J. Provost, OCA, France; E. Quemerais, SA, France; T. Rashba, MPA, Germany; J. Raymond, CFA, USA; M. Rieutord, OMP, France; E. Robbrecht, ROB, Belgium; T. Roudier, Tarbes, France; JP Rozelot, Grasse, France; V. Semikov, Izmiran, Russia; D. Socker, NRL, USA; S. Talon, Université Montréal, Canada; S. Solanki, MPI, Germany; M. Thompson, U Sheffield, England; A. Vourlidas, NRL, USA; JP Zahn, Observatoire Paris, France; A. Zhukov, ROB, Belgium.

We would like to thank all of them warmly and more specifically A. Bonanno, A. S. Brun, B. Dintrans, I. Lopes, S. Mathis, JP Rozelot, S. Talon for some very crucial inputs on the science of DynaMICCS. We have also largely shared the enthusiasm and expertise of the ALCATEL team during the preparation of the Cosmic Vision plan: B. Huet, D. Rebuffat, X. Roser, P. Terrenoire.

Open Access This article is distributed under the terms of the Creative Commons Attribution Noncommercial License which permits any noncommercial use, distribution, and reproduction in any medium, provided the original author(s) and source are credited.

\section{References}

1. Turck-Chièze, S., et al.: The magnetism of the solar interior for a complete MHD solar vision ESA-SP 588, 39th ESLAB symposium, p. 193 (2005); Turck-Chièze S. et al.: ESA-SP 624, SoHO 18/GONG 2006/HELAS I, Beyond the spherical Sun, p. 24 (2006)

2. Vorontsov, S., et al.: Helioseismic measurement of solar torsional oscillations. Science 296, 101 (2002)

3. Brummell, N., Cline, K., Cattaneo, F.: Penetration and overshooting in turbulent compressible convection. MNRAS 329, 73 (2002)

4. Turck-Chièze, S., et al.: Solar neutrino emission deduced from an helioseismic model. ApJ 555, L69 (2001)

5. Couvidat, S., Turck-Chièze, S. Kosovichev, A.: Solar seismic models and the neutrino predictions. ApJ 599, 1434 (2003)

6. Turck-Chièze, S., et al.: Looking for gravity-mode multiplets with the GOLF experiment aboard SOHO. ApJ 604, 455, 608, 610 (2004)

7. Garcia, R.A., et al.: Tracking solar gravity modes: the dynamics of the solar core. Science 316, 1591 (2007)

8. Mathur, S., Eff-Darwich, A., Garcia, R.A., Turck-Chièze, S.: Sensitivity of helioseismic gravity modes to the dynamics of the solar core. A\&A 484, 517 (2008) 
9. Mathur, S., Turck-Chièze, S., Couvidat, S ., Garcia, R.: On the characteristics of the solar gravity mode frequencies. ApJ 668, 594 (2007)

10. Zahn, J.-P.: Circulation and turbulence in rotating stars. A\&A 265, 115 (1992)

11. Maeder, A., Zahn, J.-P.: Stellar evolution with rotation. III. Meridional circulation with $\mu$ gradients and non-stationarity. A\&A 334, 1000 (1998)

12. Palacios, A., Nghiem, P., Turck-Chièze, S.: Macroscopic and microscopic behaviours of a rotating Sun. ApJ. (2008, submitted)

13. Charbonneau, P., MacGregor, K.B.: Angular momentum transport in magnetized stellar radiative zones. II. The Solar Spin-down. ApJ 417, 762 (1993)

14. Gough, D.O., Mc Intyre, M.E.: Inevitability of a magnetic field in the Sun's radiative interior. Nature 394, 755 (1998)

15. Talon, S.: Waves transport in stratified media. In: Rieutord, M., Dubrulle, B. (eds.) Stellar Fluid Dynamics and Numerical Simulations: from the Sun to Neutron Stars, vol. 21, p. 105. EAS pub. series (2006)

16. Mathis, S., Zahn, J.-P.: Transport and mixing in the radiation zones of rotating stars. I. Hydrodynamical processes. A\&A 425, 229 (2004)

17. Mathis, S., Zahn, J.-P.: Transport and mixing in the radiation zones of rotating stars. II. Axisymmetric magnetic field. A\&A 440, 653 (2005)

18. Mathis, S., Decressin, T., Palacios, A., et al.: Meridional circulation in the radiation zones of rotating stars: Origins, behaviors and consequences on stellar evolution. Astron. Notes 328, 10, 1062 (2007)

19. Brun, A.S., Jouve, L.: Global models of the magnetic sun, in waves and oscillations in the solar atmosphere: heating and magneto-seismology. In: Mendoza-Briceno, C., Erdelyi, R. (eds.) Proc. IAU Symp. 247 p. 33 (2008)

20. Parker, E.N.: A solar dynamo surface wave at the interface between convection and nonuniform rotation. ApJ 408, 707 (1993)

21. Rempel, M.: Thermal properties of magnetic flux tubes. II. Storage of flux in the solar overshoot region. A\&A 397, 1097 (2003)

22. Brainwaith, J., Spruit, H.C.: A fossil origin for the magnetic field in A stars and white dwarfs. Nature 431, 819 (2004)

23. Spiegel, E.A., Zahn, J.-P.: The solar tachocline. A\&A 265, 106 (1992)

24. Elliott, J.: Aspects of the solar tachocline. A\&A 327, 1222 (1997)

25. Dikpati, M., Cally, P.S., Gilman, P.A.: Linear analysis and nonlinear evolution of twodimensional global magnetohydrodynamic instabilities in a diffusive tachocline. ApJ 610, 597 (2004)

26. Brun, A.S., Zahn, J.P.: Magnetic confinement of the solar tachocline. A\&A 457, 665 (2006)

27. Lefebvre, S., Kosovichev, A.: Changes in the subsurface stratification of the sun with the 11-year activity cycle. ApJ 633, L149 (2005)

28. Lefebvre, S., Nghiem, P.A.P., Turck-Chièze, S.: Impact of a radius and composition variation on the solar subsurface layers stratification. ApJ (2008, accepted)

29. Priest, E.R.: Heating the solar corona by magnetic reconnection. Ap \&SS 264, 77 (1999)

30. Withbroe, G.L.: The temperature structure, mass, and energy flow in the corona and inner solar wind. ApJ 325, 442 (1988)

31. Cranmer, S.R., Field, G.B., Kohl, J.L.: Spectroscopic constraints on models of ion cyclotron resonance heating in the polar solar corona and high-speed solar wind. ApJ 518, 937 (1999)

32. Marsch, E.: Cyclotron heating of the solar corona. Ap \&SS 264, 63 (1999)

33. Peter, H, Gudiksen, B.V., Nordlund, A.: Coronal heating through braiding of magnetic field lines synthesized coronal EUV emission and magnetic structure. ESA-SP 596, 14 (2005)

34. Klimchuk, J.A.: On solving the coronal heating problem. Sol. Phys. 234, 41 (2006)

35. Koutchmy, S., et al.: The August 11th, 1999 CME. A \&A 420, 709 (2004)

36. Lin, H., Penn, M.J., Tomczyk, S.: A new precise measurement of the coronal magnetic field strength. ApJ 541, L83 (2000)

37. Lin, H., Kuhn, J.R., Coulter, R.: Coronal magnetic field measurements. ApJ 613, L177 (2004)

38. Turck-Chièze, S., Couvidat, S., Piau, L., Ferguson, E.: Surprising Sun: A new step towards a complete picture? PRL., 211102 (2004); Turck-Chièze, S., et al.: How does helioseismology constrain solar neutrino properties? Nucl. Phys. Suppl. 143, 35; Solar models and solar 
neutrinos. Nucl. Phys. Suppl. 145, 17 (2005); Turck-Chièze, S., Talon, S.: The dynamics of the solar radiative zone. Adv. Space. Res. 41, 855 (2008)

39. Bonanno, A., Schlattl, H.: Helioseismic constraints on the time evolution of the fine structure constant. SA-SP617E, 37B (2006)

40. Lopes, I., Silk, J., Hansen, S.: Helioseismology as a new constraint on supersymmetric dark matter. MNRAS 331(2), 361-368 (2002)

41. Lopes, I., Silk, J.: Solar neutrinos: probing the quasi-isothermal solar core produced by supersymmetric dark matter particles. PRL 88(15), id. 151303 (2002)

42. Rozelot, J.P., Pireaux, S., Lefebvre, S., Ajabshirizadeh, A.: Solar rotation and gravitational moments: some astrophysical outcomes. In: SOHO 14/GONG 2004 Workshop, ESA SP-559, $606(2004)$

43. Lefebvre, S., Rozelot, J.P., Kosovichev, A.: The change of solar shape in time and depth. Some consequences for space climate. Adv. Space. Res. 40, 1000 (2007)

44. Armstrong, J., Kuhn, J.R.: Interpreting the solar limb shape distortions. ApJ 525, 533 (1999)

45. Godier, S., Rozelot, J.P.: Relationships between the quadrupole moment and the internal layers of the sun. A\&A 350, 310 (1999)

46. Lefebvre, S., Kosovichev, A.G., Rozelot, J-P.: The change of solar shape in time and depth. Some consequences for space climate. Adv. Space. Res. 40, 1000 (2007)

47. Lefebvre, S., Kosovichev, A.G., Nghiem, P., Turck-Chièze, S., Rozelot, J.P.: Cyclic variability of the seismic solar radius from SOHO/MDI and related physics. In: SOHO 18 / GONG 2006 / HELAS I - Conference, Shefield, U.K., August 7-11 2006. Beyond the Spherical Sun: A New Era of Helio- and Asteroseismology. ESA SP-624, CDROM, p. 9.1 (2006)

48. Pireaux, S., Rozelot, J.P.: Cyclic variability of the seismic solar radius from SOHO/MDI and related physics. Astron. \& Space Science 284, 1159 (2003)

49. Fröhlich, C.: Solar irradiance variability since 1978. Space Sci. Rev. 125, 53 (2006)

50. Moberg, A., Sonechkin, D.M., Holmgren, K., Datsenko, N.M., Karlén, W.: Highly variable Northern Hemisphere temperatures reconstructed from low- and high-resolution proxy data. Nature 433(7026), 613 (2005)

51. Dikpati, M., Gilman, P.: Simulating and predicting solar cycles using a flux-transport dynamo. ApJ 649, 498 (2006)

52. Haigh, J.D., Lockwood, M., Giampapa, M.S.: In: Rüedi, I., Güdel, M., Schmutz, W. (eds.) The sun, solar analogs and the climate. Saas Fee Course 34, Springer (2005)

53. Woods, T.: Simulating and predicting solar cycles using a flux-transport dynamo. Eparvier. Adv. Space Res. 37, 219 (2006)

54. Lilensten, J., Dudok de Wit, T., Kretzschmar, M., et al.: Review on the solar spectral variability in the EUV for space weather purposes. Ann. Geoph. 26, 269 (2008)

55. Kretzschmar, M. Lilensten, J., Aboudarham, J.: Retrieving the solar EUV spectral irradiance from the observation of 6 lines. Adv. Space Res. 37, 341 (2006)

56. Dudok de Wit, T., Lilensten, J., Aboudarham, J., et al.: Retrieving the solar EUV spectrum from a reduced set of spectral lines. Ann. Geoph. 23, 3055 (2005)

57. Talon, S., Charbonnel, C.: Hydrodynamical stellar models including rotation, internal gravity waves, and atomic diffusion. I. Formalism and tests on Pop I dwarfs. A\&A 440981 (2005)

58. Turck-Chièze, S. et al.: GOLF - NG spectrometer, a space prototype for studying the dynamics of the deep solar interior. Adv. Space Res. 38, 1812 (2006)

59. Turck-Chièze, S., Carton, P.H., Mathur, S. et al.: Laboratory performances of the solar multichannel resonant scatteringspectrometer prototype of the GOLF-New Generation instrument. Astron. Nachr. 329, 521 (2008)

60. Thuillier, G., Dewitte, S., Schmutz, W.: The PICARD mission: scientific objectives and status of development. Adv. Space Res. 26, 1792 (2006)

61. Cacciani, A., Fofi, M.: The magneto-optical filter. II - Velocity field measurements. Sol. Phys. 59, 179 (1978)

62. Jefferies, et al.: Magnetoacoustic portals and the basal heating of the solar chromosphere. ApJ 648, 151 (2006)

63. Ruzmaikin, A., Moynihan, P.I., Vaughan, A.H., Cacciani, A.: Magnetoacoustic portals and the basal heating of the solar chromosphere. SPIE, vol. 3442, pp. 115-124 (1998)

64. Haberreiter, M., Krivova, N.A., Schmutz, W., Wenzler, T.: Reconstruction of the solar UV irradiance back to 1974. Adv. Space Res. 35, 365 (2005) 
65. Dewitte, S., Crommelynck, D., Joukoff, A.: Total solar irradiance observations from DIARAD/VIRGO. J. Geophys. Res. 109, A2 (2004)

66. Rottman, G.J., et al.: SORCE solar UV irradiance results. Adv. Space. Res. 37, 201 (2006)

67. Hochedez, J.F., Schmutz, W., Stockman, Y., et al.: LYRA, a solar UV radiometer on Proba2. Adv. Space Res. 37, 303 (2006)

68. Vivès, S., Lamy, P., et al.: Formation flying applied to solar coronal diagnostics: the ASPIICS coronagraph. Proc. SPIE, 6265 (2006)

69. Harrison, R.A., et al.: Formation flying applied to solar coronal diagnostics: the ASPIICS coronagraph. Sol. Phys. 247, 171 (2008) 